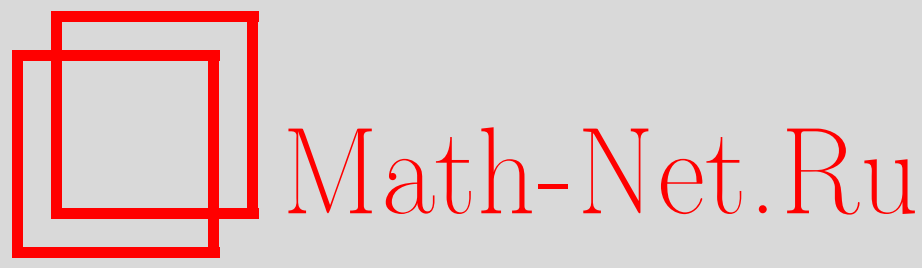

В. Н. Цыпышев, Вторая координатная последовательность линейной рекурренты максимального периода над собственным кольцом Галуа нечетной характеристики, Дискрет. матем., 2016, том 28, выпуск 1, 123-149

DOI: https://doi.org/10.4213/dm1362

Использование Общероссийского математического портала Math-Net.Ru подразумевает, что вы прочитали и согласны с пользовательским соглашением http://www . mathnet.ru/rus/agreement

Параметры загрузки:

IP: 52.6 .47 .48

26 апреля 2023 г., 17:30:06

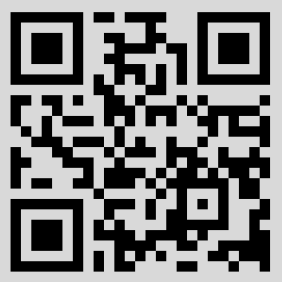




\title{
Вторая координатная последовательность линейной рекурренты максимального периода над собственным кольцом Галуа нечетной характеристики
}

\author{
() 2016 г. В. Н. Цыпышев*
}

\begin{abstract}
Под собственным кольцом Галуа понимается кольцо Галуа, отличное от поля и от кольца вычетов.

В работе описываются делители и кратные минимального многочлена второй $p$-адической координатной последовательности линейной рекуррентной последовательности максимального периода (ЛРП МП) над собственным кольцом Галуа нечетной характеристики и их связь с начальным вектором этой ЛРП.

В качестве следствий получены нетривиальные верхняя и нижняя оценки ранга второй координатной последовательности ЛРП МП над собственным кольцом Галуа нечетной характеристики.
\end{abstract}

Ключевые слова: Кольцо Галуа, линейная рекуррентная последовательность, координатная последовательность, оценка ранга

\section{1. Введение}

Пусть $R=G R\left(q^{n}, p^{n}\right)$ - кольцо Галуа $[12,13], q=p^{r}, p-$ простое число, $u$ - линейная рекуррента полного периода над кольцом $R$ с характеристическим многочленом Галуа $F(x)$ степени $m$, т. е. Т( $u)=\mathrm{T}(\bar{u}) \cdot p^{n-1}$, где Т( $\left.u\right)-$ период ЛРП $u$, а $\bar{u}-$ результат приведения каждого знака ЛРП $u$ по модулю $p R$ [6].

Пусть $S=G R\left(Q^{n}, p^{n}\right)$, где $Q=q^{m}$, - кольцо разложения многочлена $F(x), \theta-$ корень $F(x)$ в кольце $S$. Тогда [5] существует единственная константа $\xi \in S$, для которой

$$
u(i)=\operatorname{Tr}_{R}^{S}\left(\xi \theta^{i}\right), i \in \mathbb{N}_{0},
$$

где $\operatorname{Tr}_{R}^{S}(x)=\sum_{\sigma \in \operatorname{Aut}(S / R)} x^{\sigma}-$ функция $с л е д$ из кольца $S$ в кольцо $R$.

Известно, что произвольный элемент $s \in S$ однозначно представляется в виде

$$
s=\sum_{i=0}^{n-1} \gamma_{i}(s) p^{i}, \gamma_{i}(s) \in \Gamma(S), i=0, \ldots, n-1,
$$

* Место работы: Российский государственный социальный университет, e-mail: tsypyschev@yandex.ru 
где $\Gamma(S)=\left\{x \in S \mid x^{Q}=x\right\}-p$-адическое координатное множество кольца $S$ (система представителей Тейхмюллера).

Множество $\Gamma(S)$ с операциями $\oplus: x \oplus y=(x+y)^{Q^{n-1}}$ и $\otimes: x \otimes y=x y$ является полем $G F(Q)$. Поле $\Gamma(S)$ содержит в качестве подполя множество $\Gamma(R)=$ $\left\{x \in R \mid x^{q}=x\right\}-p$-адическое координатное множество кольца $R$, на котором аналогичным образом определяются операции сложения и умножения.

Известно $[12,13]$, что группа $\operatorname{Aut}(S / R)$ - циклическая и порождается автоморфизмом Фробениуса $\rho$, который на элементе $s \in S$ вида (1.2) действует следующим образом:

$$
\rho(s)=\sum_{i=0}^{n-1} \gamma_{i}(s)^{q} p^{i} .
$$

Представление, аналогичное представлению (1.2), справедливо также для элементов кольца $R$. При этом каждая последовательность $u$ над кольцом $R$ однозначно определяет $n$ координатных р-адических последователъностей $u_{l}(i)=\gamma_{l}(u(i))$, $i \in \mathbb{N}_{0}, l=1, \ldots, n-1$, над полем $(\Gamma(R), \oplus, \cdot)$.

Отметим, что $p$-адическое координатное множество является важнейшим частным случаем произвольного координатного множества $K$, которое определяется как полная система представителей классов вычетов по модулю $p R$.

Для выработки псевдослучайных последовательностей на основе линейной рекурренты над кольцом Галуа может применяться способ, состоящий в выборе одного или нескольких старших $p$-адических разрядов этой последовательности.

В этой связи представляет интерес нахождение минимальных многочленов и либо точных значений рангов последовательностей $\gamma_{l}(u), l=1, \ldots, n-1$, либо их нетривиальных оценок.

В [11] получены нижние и верхние оценки рангов координатных последовательностей линейных рекуррент максимального периода над примарными кольцами вычетов, а также минимальные многочлены координатных последовательностей некоторых классов таких линейных рекуррент.

Кроме того, в работе [11] получены минимальные многочлены последовательностей $u_{0}$ и $u_{1}$ над собственным кольцом Галуа в $p$-адическом координатном множестве. В [3] получены минимальный многочлен и ранг первой координатной последовательности линейной рекурренты $u$ над собственным кольцом Галуа в произвольном координатном множестве.

Далее, в [10] найдены точные значения ранга второй координатной последовательности линейной рекурренты $u$ над кольцом вычетов $\mathbb{Z}_{2^{n}}$ с минимальным многочленом Галуа степени $m$, не меньшей пяти, периода $2^{m}-1$ в зависимости от начального вектора этой рекурренты.

Ниже мы найдем многочлены над полем $\Gamma(R)$, которые соответственно делят и делятся на минимальный многочлен второй координатной последовательности линейной рекурренты $u$ в $p$-адическом координатном множестве при $p \geqslant 5$, и найдем, таким образом, оценки снизу и сверху для ранга этой последовательности. Результаты данной работы опубликованы в тезисах [15], [16]. 
Пусть $M, w \in \mathbb{N}$. Обозначим через $\mathcal{I}(M, w)$ множество таких векторов $\vec{\jmath}=$ $\left(j_{1}, \ldots, j_{M}\right), 0 \leqslant j_{l}, \ldots, j_{M} \leqslant p-1$, что $\sum_{l=1}^{M} j_{l}=w$, и через $\left\{\begin{array}{c}M \\ w\end{array}\right\}$ обозначим мощность множества $\mathcal{I}(M, w)$. Отметим, что $\left\{\begin{array}{c}M \\ w\end{array}\right\}$ есть число заполнений $M$ различных ячеек $w$ одинаковыми предметами, при условии, что в каждый ящик попадает не более $p-1$ предметов.

Справедливы равенства [7, с. 215]:

$$
\left\{\begin{array}{c}
M \\
w
\end{array}\right\}=\sum_{s=0}^{\min \{w,(M-w) / p\}}(-1)^{s}\left(\begin{array}{c}
w \\
s
\end{array}\right)\left(\begin{array}{c}
M+w-p s-1 \\
M-1
\end{array}\right),
$$

если $0 \leqslant w \leqslant M(p-1)$, и

$$
\left\{\begin{array}{l}
M \\
w
\end{array}\right\}=0
$$

в противном случае.

Всюду далее будем считать, что $\mathcal{N}=\left\{\begin{array}{c}m \\ p\end{array}\right\}$, где $m=\operatorname{deg} F(x)$, и что все векторы $\vec{j}^{(1)}, \ldots, \mathfrak{j}^{(\mathcal{N})}$ из множества $\mathcal{I}(m, p)$ упорядочены в лексикографическом порядке.

Напомним, что всюду далее $p \geqslant 5$.

Основными результатами настоящей работы являются теорема 3.1 , а также следствия из нее.

\section{2. Предварительные результаты}

Лемма 2.1. Пусть $M \geqslant 1, K_{1}, \ldots, K_{M} \in \mathbb{N}_{0}, K=K_{1}+\cdots+K_{M}, E-$ максимальный показатель степени простого числа $p$, при котором $p^{E}$ делит полиномиальный коэффичиент $\frac{K !}{K_{1} ! \cdots K_{M} !}$.

Пусть $\nu_{l}(x)$, где $x, l \in \mathbb{N}_{0}$, обозначает l-й разряд $p$-ичного разложения числа $x, a$ $w(x)-p$-ичный вес числа $x$, m.е. сумма всех разрядов р-ичного разложения этого числа. Справедливы следующие утверждения.

(a) $E=\frac{w\left(K_{1}\right)+\ldots+w\left(K_{M}\right)-w(K)}{p-1}$.

(b)

$$
\frac{K !}{K_{1} ! \cdots K_{M} !} \equiv(p !)^{E} \cdot \prod_{l \geqslant 0} \frac{\nu_{l}(K) !}{\nu_{l}\left(K_{1}\right) ! \cdots \nu_{l}\left(K_{M}\right) !}\left(\bmod p^{E+1}\right) .
$$

(c) Пусть $K=\sum_{i=0}^{\kappa} \nu_{i}(K) p^{i}, \epsilon=\|K !\|_{p}=\max \left\{\epsilon \in \mathbb{N}_{0} \quad: \quad p^{\epsilon} \mid K !\right\}=[K / p]+$ $\left[K / p^{2}\right]+\ldots$ Тогда

$$
\begin{aligned}
K ! \equiv(p !)^{\epsilon}\left(\nu_{0}(K) ! \cdots\right. & \nu_{\kappa}(K) !+ \\
& \left.p \sum_{i=0}^{\kappa-1} \sigma_{\nu_{i}(K)-1}\left(1, \ldots, \nu_{i}(K)\right) \nu_{i+1}(K) \prod_{\substack{j=0 \\
j \neq i}}^{\kappa} \nu_{j}(K) !\right)\left(\bmod p^{\epsilon+2}\right),
\end{aligned}
$$

где $\sigma_{r}\left(y_{1}, \ldots, y_{s}\right)$ - симметрическая функиия степени $r$ от переменных $y_{1}, \ldots, y_{s}$, $\sigma_{-1}=0, \sigma_{0}=1$. 
(d) Пусть $K_{l}=\sum_{i=0}^{\kappa} \nu_{i}\left(K_{l}\right) p^{i}, l=1, \ldots, M$. Тогда

$$
\begin{aligned}
\frac{K !}{K_{1} ! \cdots K_{M} !} p^{\overline{\overline{E+2}}} & \frac{\prod_{j=0}^{\kappa} \nu_{j}(K) !+p \sum_{i=0}^{\kappa-1} \sigma_{\nu_{i}(K)-1}\left(1, \ldots, \nu_{i}(K)\right) \nu_{i+1}(K) \prod_{\substack{j=0 \\
j \neq i}}^{\kappa} \nu_{j}(K) !}{p^{\overline{\overline{E+}}}(p !)^{E}} \frac{\prod_{l=1}^{M}\left(\prod_{j=0}^{\kappa} \nu_{j}\left(K_{l}\right) !+p \sum_{i=0}^{\kappa-1} \sigma_{\nu_{i}\left(K_{l}\right)-1}\left(1, \ldots, \nu_{i}\left(K_{l}\right)\right) \nu_{i+1}\left(K_{l}\right) \prod_{\substack{j=0 \\
j \neq i}}^{\kappa} \nu_{j}\left(K_{l}\right) !\right)}{} .
\end{aligned}
$$

Доказательство. (а) Следует из [4, лемма 6.39].

(b) Следует из [2, лемма 1(б)].

(c) Имеет место цепочка соотношений

$$
\begin{aligned}
& K !=\left(\prod_{t=1}^{[K / p]}((p(t-1)+1) \cdots(p(t-1)+p-1) p t)\right) \times \\
& \times(p[K / p]+1) \cdots\left(p[K / p]+\nu_{0}(K)\right) \underset{p^{\epsilon+2}}{\equiv} \\
& p^{\overline{\epsilon+2}}\left(\prod_{t=1}^{[K / p]}\left(\left((p-1) !+p(t-1) \sigma_{p-2}(1, \ldots, p-1)\right) p t\right)\right) \times \\
& \quad \times\left(\nu_{0}(K) !+p[K / p] \sigma_{\nu_{0}}(K)-1\left(1, \ldots, \nu_{0}(K)\right) \underset{p^{\epsilon+2}}{\equiv}\right. \\
& \equiv(p !)^{[K / p]} \cdot[K / p] ! \cdot\left(\nu_{0}(K) !+p \nu_{1}(K) \sigma_{\nu_{0}(K)-1}\left(1, \ldots, \nu_{0}(K)\right)\right) .
\end{aligned}
$$

Сделанные переходы справедливы, поскольку имеют место равенства

$$
\begin{aligned}
& \left(a_{1}+p b\right) \cdots\left(a_{s}+p b\right) \underset{p^{2}}{\equiv} a_{1} \cdots a_{s}+p b \sigma_{s-1}\left(a_{1}, \ldots, a_{s}\right), \\
& \sigma_{p-2}(1, \ldots, p-1)=(-1)^{p-2} \operatorname{coeff}_{x}((x-1) \cdots(x-(p-1)) \underset{\bar{p}}{\bar{E}} \\
& \overline{\bar{p}}(-1)^{p-2} \operatorname{coeff}_{x}\left(x^{p-1}-1\right)=0 \text { при } p \geqslant 3 \text {, } \\
& {[K / p] \underset{p}{\equiv} \nu_{1}(K)}
\end{aligned}
$$

Применяя проделанные выше рассуждения к параметрам $[K / p],\left[K / p^{2}\right]$ и т.д., окончательно получим:

$$
\begin{gathered}
K ! \underset{p^{\epsilon+2}}{\equiv}(p !)^{\epsilon}\left(\nu_{0}(K) !+p \nu_{1}(K) \sigma_{\nu_{0}(K)-1}\left(1, \ldots, \nu_{0}(K)\right) \cdots\right. \\
\cdots\left(\nu_{\kappa-1}(K) !+p \nu_{\kappa}(K) \sigma_{\nu_{\kappa-1}}(K)-1\left(1, \ldots, \nu_{\kappa-1}(K)\right)\right) \nu_{\kappa}(K) !
\end{gathered}
$$

Следовательно,

$$
K ! \underset{p^{\epsilon+2}}{\equiv}(p !)^{\epsilon}\left(\nu_{0}(K) ! \cdots \nu_{\kappa}(K) !+\right.
$$




$$
\begin{aligned}
& \left.+p \sum_{i=0}^{\kappa-1} \nu_{i+1}(K) \sigma_{\nu_{i}(K)-1}\left(1, \ldots, \nu_{i}(K)\right) \prod_{\substack{j=0,0, \kappa \\
j \neq i}} \nu_{j}(K) !\right) p_{p^{\epsilon+2}} \sum_{p^{\epsilon+2}}(p !)^{\epsilon}\left(\prod_{j=0}^{\kappa} \nu_{j}(K) !+p \sum_{i=0}^{\kappa-1} \nu_{i+1}(K) \sigma_{\nu_{i}(K)-1}\left(1, \ldots, \nu_{i}(K)\right) \prod_{\substack{j=0, \kappa \\
j \neq i}} \nu_{j}(K) !\right) .
\end{aligned}
$$

(d) Непосредственно следует из пункта (c) с учетом равенства

$$
E=\epsilon-\sum_{l=1}^{M} \epsilon_{l}, \text { где } \epsilon_{l}=\left\|K_{l}\right\|_{p} .
$$

Отметим, что утверждения леммы 2.1 справедливы также при $p=3$.

Лемма 2.2. Пусть $Q=p^{t}, t=r m>2$. Тогда все решения системь

$$
\left\{\begin{array}{l}
\vec{\jmath} \in\{0, \ldots, Q-1\}^{M}, \\
\sum_{l=1}^{M} j_{l}=Q, \\
\left\|\frac{Q !}{\prod_{l=1}^{M} j_{l} !}\right\|_{p}=2
\end{array}\right.
$$

имеют вид

$$
\begin{gathered}
\vec{\jmath}=\left(\lambda_{1} p^{t-2}+\zeta_{1} p^{t-1}, \ldots, \lambda_{M} p^{t-2}+\zeta_{M} p^{t-1}\right), \\
\vec{\lambda} \in \mathcal{I}(M, p), \quad \vec{\zeta} \in \mathcal{I}(M, p-1) .
\end{gathered}
$$

Доказательство. Рассмотрим систему (2.1). Пусть

$$
j_{l}=\sum_{\kappa=0}^{t-1} j_{l}^{(\kappa)} p^{\kappa}
$$

- $p$-ичное разложение числа $j_{l}, l=1, \ldots, M$. Тогда, с учетом леммы 2.1(a), система (2.1) перепишется в виде

$$
\left\{\begin{array}{l}
\sum_{l=1}^{M} \sum_{\kappa=0}^{t-1} j_{l}^{(\kappa)} p^{\kappa}=p^{t} \\
\sum_{l=1}^{M} \sum_{\kappa=0}^{t-1} j_{l}^{(\kappa)}=2 p-1 .
\end{array}\right.
$$

Положим

$$
d_{\kappa}=\sum_{l=1}^{M} j_{l}^{(\kappa)}
$$

Тогда получим:

$$
\left\{\begin{array}{l}
\sum_{\kappa=0}^{t-1} d_{\kappa} p^{\kappa}=p^{t} \\
\sum_{\kappa=0}^{t-1} d_{\kappa}=2 p-1
\end{array}\right.
$$


Очевидно, что набор

$$
d_{0}=\ldots=d_{t-3}=0, d_{t-2}=p, d_{t-1}=p-1
$$

является решением системы (2.3). Покажем, что других решений нет.

Если $d_{t-1} \geqslant p$, то $\sum_{\kappa=0}^{t-1} d_{\kappa} p^{\kappa}>p^{t}$, что противоречит первому уравнению системы (2.3).

Если $d_{t-1}=0$, то $\sum_{\kappa=0}^{t-2} d_{\kappa} p^{\kappa} \leqslant(2 p-1) p^{t-2}<p^{t}$, поскольку $p \geqslant 3$, и, следовательно, $2 p-1<p^{2}$.

Пусть $0<d_{t-1}<p-1$.

Напомним, что через $\operatorname{Res}(a, b), a, b \in \mathbb{N}_{0}, b \neq 0$, принято обозначать остаток от целочисленного деления $a$ на $b$, а через $\left[\frac{a}{b}\right]$ - нижнюю целую часть числа $\frac{a}{b}$.

Положим

$$
\tilde{d}_{0}=\operatorname{Res}\left(d_{0}, p\right), \tilde{d}_{\kappa}=\operatorname{Res}\left(\left(d_{\kappa}+\epsilon_{\kappa-1}\right), p\right), \kappa=0, \ldots, t-3, \tilde{d}_{t-2}=d_{t-2}+\epsilon_{t-3},
$$

где

$$
\epsilon_{-1}=0, \epsilon_{\kappa}=\left[\frac{\epsilon_{\kappa-1}+d_{\kappa}}{p}\right], \kappa=0, \ldots, t-2
$$

Тогда

$$
\sum_{\kappa=0}^{t-2} d_{\kappa} p^{\kappa}=\sum_{\kappa=0}^{t-2} \tilde{d}_{\kappa} p^{\kappa}
$$

Так как $0 \leqslant d_{\kappa}<2 p-1$, то $\epsilon_{\kappa} \in\{0,1\}, \kappa=0, \ldots, t-2$.

Из системы $(2.3)$ и определения чисел $\tilde{d}_{\kappa}, \kappa=0, \ldots, t-2$, следует система соотношений

$$
\left\{\begin{array}{l}
\sum_{\kappa=0}^{t-2} \tilde{d}_{\kappa} p^{\kappa}=p^{t-1}\left(p-d_{t-1}\right) \\
0 \leqslant \tilde{d}_{\kappa}<p, \kappa=0, \ldots, t-3 \\
0 \leqslant \tilde{d}_{t-2} \leqslant 2 p-d_{t-1}
\end{array}\right.
$$

Следовательно,

$$
\sum_{\kappa=0}^{t-3} \tilde{d}_{\kappa} p^{\kappa}+\tilde{d}_{t-2} p^{t-1}+d_{t-1} p^{t-1}=p \cdot p^{t-2}+(p-1) p^{t-1}
$$

отсюда

$$
\sum_{\kappa=0}^{t-3} \tilde{d}_{\kappa} p^{\kappa}=\left(p-1-d_{t-1}\right) p^{t-1}+\left(p-\tilde{d}_{t-2}\right) p^{t-2} .
$$

Поскольку

$$
0 \leqslant \tilde{d}_{\kappa} \leqslant p-1, \quad \kappa=0, \ldots, t-3
$$

TO

$$
\sum_{\kappa=0}^{t-3} \tilde{d}_{\kappa} p^{\kappa} \leqslant(p-1) \cdot \frac{p^{t-2}-1}{p-1}=p^{t-2}-1
$$

отсюда

$$
\left(p-1-d_{t-1}\right) p+\left(p-1-\tilde{d}_{t-2}\right)<0 .
$$


Следовательно, поскольку $1 \leqslant d_{t-1} \leqslant p-2$, имеем :

$$
(p-2) p+\left(p-1-\tilde{d}_{t-2}\right)<0,
$$

то есть

$$
\tilde{d}_{t-2}>p^{2}-3 p-1
$$

С другой стороны,

$$
0 \leqslant \tilde{d}_{t-2} \leqslant 2 p-1
$$

В силу двух предыдущих неравенств

$$
2 p-1>p^{2}-3 p-1
$$

или

$$
p<5 \text {. }
$$

Поскольку случай $p=3$ исключен из рассмотрения, получаем противоречие, говорящее о том, что случай $0<d_{t-1}<p$ невозможен.

Следовательно, набор (2.4) действительно является единственным решением системы (2.3). Отсюда следует утверждение леммы.

Определим элемент $\nabla \in \Gamma\left(\mathbb{Z}_{p^{n}}\right)$ как

$$
(p-1) ! \equiv-1-p \nabla \quad\left(\bmod p^{2}\right) .
$$

Введем в рассмотрение многочлены над кольцом $\Gamma\left(\mathbb{Z}_{p^{n}}\right)\left[x_{1}, \ldots, x_{M}\right]$, положив для краткости $\vec{x}=\left(x_{1}, \ldots, x_{M}\right)$ :

$$
W^{(Q, 1)}\left(x_{1}, \ldots, x_{M}\right)=\bigoplus_{\vec{\jmath} \in \mathcal{I}(M, p)} w_{\vec{\jmath}}^{(Q, 1)} \prod_{l=1}^{M} x_{l}^{j_{l} p^{t-1}} \in \Gamma\left(\mathbb{Z}_{p^{n}}\right)[\vec{x}],
$$

где

$$
w_{\vec{\jmath}}^{(Q, 1)}=\gamma_{0}\left(\frac{\nabla}{\prod_{l=1}^{M} j_{l} !}\right) \oplus \gamma_{1}\left(\frac{1}{\prod_{l=1}^{M} j_{l} !}\right)
$$

и

$$
W^{(Q, 2,1)}\left(x_{1}, \ldots, x_{M}\right)=\bigoplus_{s=0}^{p} \bigoplus_{\substack{\vec{\lambda} \in \mathcal{I}(M, p s), \vec{\zeta} \in \mathcal{I}(M, p-s)}} w_{\vec{\lambda}, \vec{\zeta}}^{(Q, 2,1)} \prod_{l=1}^{M} x_{l}^{p^{t-2}\left(\lambda_{l}+p \zeta_{l}\right)} \in \Gamma\left(\mathbb{Z}_{p^{n}}\right)[\vec{x}],
$$

где

а также

$$
w_{\vec{\lambda}, \vec{\zeta}}^{(Q, 2,1)}=\bigoplus_{\substack{\vec{\mu} \in \mathcal{I}(\mathcal{N}, p): \\ \sum_{i=1}^{N} \mu_{i} j_{l}^{(i)}=\lambda_{l}+p \zeta_{l}, \forall l=1, \ldots, M}} \gamma_{0}\left(\frac{1}{\prod_{i=1}^{N} \mu_{i} !\left(\prod_{l=1}^{M} j_{l}^{(i)} !\right)^{\mu_{i}}}\right)
$$

$$
W^{(Q, 2,2)}\left(x_{1}, \ldots, x_{M}\right)=\bigoplus_{\substack{\vec{\lambda} \in \mathcal{I}(M, p), \vec{\zeta} \in \mathcal{I}(M, p-1)}} w_{\vec{\lambda}, \vec{\zeta}}^{(Q, 2,2)} \prod_{l=1}^{M} x_{l}^{p^{t-2}\left(\lambda_{l}+p \zeta_{l}\right)} \in \Gamma\left(\mathbb{Z}_{p^{n}}\right)[\vec{x}],
$$

5 Дискретная математика, т. 28 № 1 
где

$$
w_{\vec{\lambda}, \vec{\zeta}}^{(Q, 2,2)}=\gamma_{0}\left(\frac{1}{\prod_{l=1}^{M} \lambda_{l} ! \zeta_{l} !}\right) .
$$

Лемма 2.3. Пусть $S=G R\left(Q^{n}, p^{n}\right), Q=q^{m}, q=p^{r}, t=r m>2$. Тогда для любых $x_{1}, \ldots, x_{M} \in \Gamma(S)$ имеет место равенство

$$
\gamma_{2}\left(x_{1}+\cdots+x_{M}\right)=W^{(Q, 1)}(\vec{x}) \oplus W^{(Q, 2,1)}(\vec{x}) \ominus W^{(Q, 2,2)}(\vec{x}) .
$$

Доказательство. Положим

$$
\sigma=x_{1}+\cdots+x_{M}
$$

и найдем $\gamma_{1}(\sigma)$ и $\gamma_{2}(\sigma)$. Имеем:

$$
\sigma^{Q}=x_{1}^{Q}+\cdots+x_{M}^{Q}+\sum_{\substack{\vec{\jmath} \in\{0, \ldots, Q-1\} M \\ j_{1}+\cdots+j_{M}=Q}} \frac{Q !}{j_{1} ! \cdots j_{M} !} x_{1}^{j_{1}} \cdots x_{M}^{j_{M}} .
$$

Заметим, что $x_{i}^{Q}=x_{i}, i=1, \ldots, M$, поскольку $x_{i} \in \Gamma(S)$. Следовательно,

$$
x_{1}^{Q}+\cdots+x_{M}^{Q}=x_{1}+\cdots+x_{M} .
$$

Теперь рассмотрим отдельно левую часть равенства $(2.24):$ так как $\sigma \underset{p}{\bar{p}} \gamma_{0}(\sigma)$, то для любого $t \geqslant 2$ выполнено сравнение $\sigma^{p^{t}} \underset{p^{3}}{\equiv} \gamma_{0}(\sigma)^{p^{t}}$. Следовательно,

$$
\sigma^{Q} \underset{\overline{p^{3}}}{\equiv} \gamma_{0}(\sigma)^{Q}=\gamma_{0}(\sigma)
$$

Выписанные соотношения справедливы, поскольку $p^{2} \mid Q=p^{t}=p^{r m}$ и $\gamma_{0}(\sigma) \in \Gamma(S)$.

Подставляя соотношения (2.25) и $(2.26)$ в $(2.24)$, получим:

$$
\gamma_{0}(\sigma) \underset{p^{3}}{\equiv} \sigma+\sum_{\substack{\vec{\jmath} \in\{0, \ldots, Q-1\} \\ \sum_{l=1}^{M} j_{l}=Q}} \frac{Q !}{\prod_{l=1}^{M} j_{l} !} \prod_{l=1}^{M} x_{l}^{j_{l}} .
$$

Введем обозначения:

$$
\tilde{W}_{1}^{(Q)}\left(x_{1}, \ldots, x_{M}\right)=\sum_{\vec{j} \in J_{1}} \frac{Q !}{\prod_{l=1}^{M} j_{l} !} \prod_{l=1}^{M} x_{l}^{j_{l}}
$$

и

$$
\tilde{W}_{2}^{(Q)}\left(x_{1}, \ldots, x_{M}\right)=\sum_{\vec{j} \in J_{2}} \frac{Q !}{\prod_{l=1}^{M} j_{l} !} \prod_{l=1}^{M} x_{l}^{j_{l}},
$$


где $J_{1}$ и $J_{2}-$ множества решений систем $(2.31)$ и (2.32) соответственно. Из (2.27) следует соотношение

$$
\tilde{W}_{1}^{(Q)}(\vec{x})+\tilde{W}_{2}^{(Q)}(\vec{x})+p \gamma_{1}(\sigma)+p^{2} \gamma_{2}(\sigma) \underset{p^{3}}{\equiv} 0 .
$$

Следовательно, для того, чтобы найти выражение для $\gamma_{2}(\sigma)$, достаточно выписать явный вид многочленов $\tilde{W}_{1}^{(Q)}(\vec{x})$ и $\tilde{W}_{2}^{(Q)}(\vec{x})$. Для этого, в свою очередь, требуется найти решения систем

и

$$
\left\{\begin{array}{l}
\vec{\jmath} \in\{0, \ldots, Q-1\}^{M}, \\
\sum_{l=1}^{M} j_{l}=Q \\
\left\|\frac{Q !}{\prod_{l=1}^{M} j_{l} !}\right\|_{p}=1
\end{array}\right.
$$

$$
\left\{\begin{array}{l}
\vec{\jmath} \in\{0, \ldots, Q-1\}^{M} \\
\sum_{l=1}^{M} j_{l}=Q \\
\left\|\frac{Q !}{\prod_{l=1}^{M} j_{l} !}\right\|_{p}=2
\end{array}\right.
$$

Хорошо известно [1, лемма 15.576$]$, что решением системы (2.31) являются только векторы вида

$$
\vec{\jmath}=\left(j_{1} p^{t-1}, \ldots, j_{M} p^{t-1}\right), \text { где } \sum_{l=1}^{M} j_{l}=p, 0 \leqslant j_{l} \leqslant p-1, l=1, \ldots, M .
$$

Отсюда с помощью леммы 2.1(d) нетрудно получить, что

$$
\tilde{W}_{1}^{(Q)}(\vec{x}) \underset{p^{3}}{\equiv} \sum_{\vec{\jmath} \in \mathcal{I}(M, p)} \frac{p !}{\prod_{l=1}^{M} j_{l} !} \prod_{l=1}^{M} x_{l}^{j_{l} p^{t-1}} .
$$

Как показывает лемма 2.2, многочлен (2.29) имеет вид

$$
\tilde{W}_{2}^{(Q)}(\vec{x})=\sum_{\substack{\vec{\lambda} \in \mathcal{I}(M, p), \vec{\zeta} \in \mathcal{I}(M, p-1)}} \frac{Q !}{\prod_{l=1}^{M}\left(\lambda_{l} p^{t-2}+\zeta_{l} p^{t-1}\right) !} \prod_{l=1}^{M} x_{l}^{p^{t-2}\left(\lambda_{l}+p \zeta_{l}\right)} .
$$

С учетом леммы 2.1(b) имеем:

$$
\tilde{W}_{2}^{(Q)}(\vec{x}) \underset{p^{3}}{\equiv} \sum_{\substack{\vec{\lambda} \in \mathcal{I}(M, p), \vec{\zeta} \in \mathcal{I}(M, p-1)}} \frac{(p !)^{2}}{\prod_{l=1}^{M} \lambda_{l} ! \zeta_{l} !} \prod_{l=1}^{M} x_{l}^{p^{t-2}\left(\lambda_{l}+p \zeta_{l}\right)} .
$$

Теперь соотношение (2.30) можно представить в виде

$$
\begin{gathered}
0 \equiv p\left(\gamma_{1}(\sigma)-\sum_{\vec{\jmath} \in \mathcal{I}(M, p)} \frac{1}{\prod_{l=1}^{M} j_{l} !} \prod_{l=1}^{M} x_{l}^{j_{l} p^{t-1}}\right)+ \\
+p^{2}\left(\gamma_{2}(\sigma)-\nabla \cdot \sum_{\vec{\jmath} \in \mathcal{I}(M, p)} \frac{1}{\prod_{l=1}^{M} j_{l} !} \prod_{l=1}^{M} x_{l}^{j_{l} p^{t-1}}+\sum_{\substack{\vec{\lambda} \in \mathcal{I}(M, p), \vec{\zeta} \in \mathcal{I}(M, p-1)}} \frac{1}{\prod_{l=1}^{M} \lambda_{l} ! \zeta_{l} !} \prod_{l=1}^{M} x_{l}^{p^{t-2}\left(\lambda_{l}+p \zeta_{l}\right)}\right) .
\end{gathered}
$$


Непосредственно из соотношения (2.37) вытекает хорошо известное равенство [11]

$$
\gamma_{1}(\sigma) \underset{p}{\equiv} W_{1}^{(Q)}\left(x_{1}, \ldots, x_{M}\right)
$$

где

$$
W_{1}^{(Q)}\left(x_{1}, \ldots, x_{M}\right)=\sum_{\vec{\jmath} \in \mathcal{I}(M, p)} \frac{1}{\prod_{l=1}^{M} j_{l} !} \prod_{l=1}^{M} x_{l}^{j_{l} p^{t-1}}
$$

Далее, соотношение (2.37) позволяет также получить выражение для $\gamma_{2}(\sigma)$, а именно:

$$
\gamma_{2}(\sigma) \underset{p}{\equiv} \nabla \cdot W_{1}^{(Q)}(\vec{x})-W_{2}^{(Q)}(\vec{x})+\gamma_{1}\left(W_{1,0}^{(Q)}(\vec{x})\right)+W_{1,1}^{(Q)}(\vec{x})
$$

где

$$
\begin{gathered}
W_{2}^{(Q)}=\sum_{\substack{\vec{\lambda} \in \mathcal{I}(M, p), \vec{\zeta} \in \mathcal{I}(M, p-1)}} \frac{1}{\prod_{l=1}^{M} \lambda_{l} ! \zeta_{l} !} \prod_{l=1}^{M} x_{l}^{p^{t-2}\left(\lambda_{l}+p \zeta_{l}\right)}, \\
W_{1,0}^{(Q)}\left(x_{1}, \ldots, x_{M}\right)=\sum_{\vec{\jmath} \in \mathcal{I}(M, p)} \gamma_{0}\left(\frac{1}{\prod_{l=1}^{M} j_{l} !}\right) \prod_{l=1}^{M} x_{l}^{j_{l} p^{t-1}}, \\
W_{1,1}^{(Q)}\left(x_{1}, \ldots, x_{M}\right)=\sum_{\vec{\jmath} \in \mathcal{I}(M, p)} \gamma_{1}\left(\frac{1}{\prod_{l=1}^{M} j_{l} !}\right) \prod_{l=1}^{M} x_{l}^{j_{l} p^{t-1}} .
\end{gathered}
$$

Применим теперь соотношения (2.38), (2.39) для того, чтобы найти явный вид выражения $\gamma_{1}\left(W_{1,0}^{(Q)}\right)$ в правой части равенства $(2.40)$. Для этого представим множество $\mathcal{I}(M, p)$ в виде

$$
\mathcal{I}(M, p)=\left\{\left(j_{1}^{(1)}, \ldots, j_{M}^{(1)}\right), \ldots,\left(j_{1}^{(\mathcal{N})}, \ldots, j_{M}^{(\mathcal{N})}\right)\right\}
$$

Тогда

$$
W_{1,0}^{(Q)}(\vec{x})=\sum_{i=1}^{\mathcal{N}} \gamma_{0}\left(\frac{1}{\prod_{l=1}^{M} j_{l}^{(i)} !}\right) \prod_{l=1}^{M} x_{l}^{j_{l}^{(i)}} p^{t-1}
$$

и

$$
\begin{gathered}
=\bigoplus_{\vec{\mu} \in \mathcal{I}(\mathcal{N}, p)}\left(\gamma_{0}\left(\frac{1}{\prod_{i=1}^{\mathcal{N}} \mu_{i} !}\right) \prod_{i=1}^{\mathcal{N}} \gamma_{0}\left(\frac{1}{\prod_{l=1}^{M} j_{l}^{(i)} !}\right)^{\mu_{i} p^{t-1}} \prod_{i=1}^{\mathcal{N}} \prod_{l=1}^{M}\left(x_{l, 0}^{(Q)}(\vec{x})\right)=\right. \\
=\bigoplus_{\vec{\mu} \in \mathcal{I}(\mathcal{N}, p)} \gamma_{0}^{(i)}\left(\frac{1}{\prod_{i=1}^{\mathcal{N}} \mu_{i} !\left(\prod_{l=1}^{M} j_{l}^{(i)} !\right)^{\mu_{i}}}\right) \prod_{i=1}^{\mathcal{N}} \prod_{l=1}^{M} x_{l}^{p^{t-2}\left(j_{l}^{(i)} \mu_{i}\right)}= \\
=\bigoplus_{\vec{\mu} \in \mathcal{I}(\mathcal{N}, p)} \gamma_{0}\left(\frac{1}{\prod_{i=1}^{\mathcal{N}} \mu_{i} !\left(\prod_{l=1}^{M} j_{l}^{(i)} !\right)^{\mu_{i}}}\right) \prod_{l=1}^{M} x_{l}^{p^{t-2} \sum_{i=1}^{\mathcal{N}} \mu_{i} j_{l}^{(i)}} .
\end{gathered}
$$


Сделанные переходы законны, поскольку, во-первых,

$$
\gamma_{0}\left(\frac{1}{\prod_{l=1}^{M} j_{l}^{(i) !}}\right) \in \Gamma\left(\mathbb{Z}_{p^{n}}\right)
$$

и, следовательно,

$$
\gamma_{0}\left(\frac{1}{\prod_{l=1}^{M} j_{l}^{(i)} !}\right)^{p}=\gamma_{0}\left(\frac{1}{\prod_{l=1}^{M} j_{l}^{(i)} !}\right)
$$

и, во-вторых, поскольку при всех $i=1, \ldots, \mathcal{N}, l=1, \ldots, M$

$$
\left(x_{l}^{j_{l}^{(i)} p^{t-1}}\right)^{\mu_{i} p^{t-1}}=x_{l}^{\mu_{i} j_{l}^{(i)} p^{2 t-2}}=\left(x_{l}^{\mu_{i} j_{l}^{(i)} p^{t-2}}\right)^{p^{t}}=x_{l}^{\mu_{i} j_{l}^{(i)} p^{t-2}} .
$$

Пусть $b_{\vec{\mu}, l}=\sum_{i=1}^{\mathcal{N}} \mu_{i} j_{l}^{(i)}$. Тогда

$$
\sum_{l=1}^{M} b_{\vec{\mu}, l}=\sum_{l=1}^{M} \sum_{i=1}^{\mathcal{N}} \mu_{i} j_{l}^{(i)}=\sum_{i=1}^{\mathcal{N}} \sum_{l=1}^{M} j_{l}^{(i)} \mu_{i}=\sum_{i=1}^{\mathcal{N}} p \mu_{i}=p^{2} .
$$

Кроме того, $b_{\vec{\mu}, l} \in\{0, \ldots, p(p-1)\}$. Следовательно, $b_{\vec{\mu}, l}$ однозначно представляется в виде

$$
b_{\vec{\mu}, l}=\lambda_{\vec{\mu}, l}+p \zeta_{\vec{\mu}, l}, \lambda_{\vec{\mu}, l}, \zeta_{\vec{\mu}, l} \in\{0, \ldots, p-1\}, l=1, \ldots, M .
$$

Изучим, в каких пределах могут изменяться значения $\sum_{l=1}^{M} \lambda_{\vec{\mu}, l}$ и $\sum_{l=1}^{M} \zeta_{\vec{\mu}, l}$. Пусть $\sum_{l=1}^{M} \lambda_{\vec{\mu}, l}=\alpha$. Тогда $p \sum_{l=1}^{M} \zeta_{\vec{\mu}, l}=p^{2}-\alpha$. Следовательно, $\alpha=p s, s=0, \ldots, p$, отсюда $\sum_{l=1}^{M} \zeta_{\vec{\mu}, l}=p-s$.

Таким образом, равенство (2.44) можно продолжить:

$$
\left.\gamma_{1}\left(W_{1,0}^{(Q)}(\vec{x})\right)=\bigoplus_{s=0}^{p} \bigoplus_{\substack{\vec{\lambda} \in \mathcal{I}(M, p s), \vec{\zeta} \in \mathcal{I}(M, p-s)}} \bigoplus_{\substack{\vec{\mu} \in \mathcal{I}(\mathcal{N}, p): \\ \sum_{i=1}^{\mathcal{N}} \mu_{i} j_{l}^{(i)}=\lambda_{l}+p \zeta_{l}, \forall l=1, \ldots, M}} \gamma_{0}\left(\frac{1}{\prod_{i=1}^{\mathcal{N}} \mu_{i} !\left(\prod_{l=1}^{M} j_{l}^{(i)}\right)^{\mu_{i}}}\right)\right) \prod_{l=1}^{M} x_{l}^{p^{t-2}\left(\lambda_{l}+p \zeta_{l}\right)} .
$$

Подставляя теперь соотношения (2.39), (2.41), (2.43), (2.45) в равенство (2.40), получим:

$$
\begin{aligned}
& \gamma_{2}(\sigma)=\bigoplus_{\vec{\jmath} \in \mathcal{I}(M, p)}\left(\gamma_{0}\left(\frac{\nabla}{\prod_{l=1}^{M} j_{l} !}\right) \oplus \gamma_{1}\left(\frac{1}{\prod_{l=1}^{M} j_{l} !}\right)\right) \prod_{l=1}^{M} x_{l}^{j_{l} p^{t-1}} \oplus \\
& \oplus \bigoplus_{s=0}^{p} \bigoplus_{\substack{\vec{\lambda} \in \mathcal{I}(M, p s), \vec{\zeta} \in \mathcal{I}(M, p-s)}}\left(\sum_{\substack{\vec{\mu} \in \mathcal{I}(\mathcal{N}, p): \\
\sum_{i=1}^{\mathcal{N}} \mu_{i} j_{l}^{(i)}=\lambda_{l}+p \zeta_{l}, l=1, \ldots, M}}^{\bigoplus} \gamma_{0}\left(\frac{1}{\prod_{i=1}^{\mathcal{N}} \mu_{i} !\left(\prod_{l=1}^{M} j_{l}^{(i)}\right)^{\mu_{i}}}\right)\right) \prod_{l=1}^{M} x_{l}^{p^{t-2}\left(\lambda_{l}+p \zeta_{l}\right)} \ominus \\
& \ominus \bigoplus_{\substack{\vec{\lambda} \in \mathcal{I}(M, p), \vec{\zeta} \in \mathcal{I}(M, p-1)}} \gamma_{0}\left(\frac{1}{\prod_{l=1}^{M} \lambda_{l} ! \zeta_{l} !}\right) \prod_{l=1}^{M} x_{l}^{p^{t-2}\left(\lambda_{l}+p \zeta_{l}\right)},
\end{aligned}
$$

что и утверждалось. 
Лемма 2.4. Пусть последовательности $v, w \in \Gamma\left(\mathbb{Z}_{p^{n}}\right)^{\infty}$ определены равенствами $v(i)=\gamma_{0}(i), w(i)=\gamma_{1}(i), i \in \mathbb{N}_{0}$.

Тогда минимальный многочлен $m_{v}(x)$ последовательности $v$ над полем $\Gamma\left(\mathbb{Z}_{p^{n}}\right)$ ecms

$$
m_{v}(x)=(x \ominus e)^{2},
$$

а минимальный многочлен $m_{w}(x)$ последовательности $w$ над полем $\Gamma\left(\mathbb{Z}_{p^{n}}\right)$ есть

$$
m_{w}(x)=(x \ominus e)^{p+1} .
$$

Доказательство. Докажем равенство (2.47):

$$
\forall i \in \mathbb{N}_{0} \gamma_{0}((i+1)) \ominus \gamma_{0}(i) \underset{p}{\equiv}(i+1)^{p}-i^{p} \underset{p}{\equiv} i^{p}+1-i^{p}=1 .
$$

Отсюда следует первое утверждение леммы.

Второе доказывается аналогично.

Также нам потребуются следующие хорошо известные факты.

Лемма 2.5. Пусть $P$ - поле, содержащее произвольные элементы $a_{1}, \ldots, a_{\kappa}, u$ различные элементы $\eta_{1}, \ldots, \eta_{\kappa}$, и пусть каэдый знак последовательности и имеет вид

$$
u(i)=\sum_{k=1}^{\kappa} a_{k} \eta_{k}^{i}, i \geqslant 0 .
$$

Тогда минимальный многочлен $m_{u}(x)$ последовательности и определяется равенством

$$
m_{u}(x)=\prod_{\substack{k \in\{1, \ldots, \kappa\}: \\ a_{k} \neq 0}}\left(x-\eta_{k}\right) .
$$

Далее, пусть каждый знак последовательности $v \in P^{\infty}$ имеет вид

$$
v(i)=\left(\begin{array}{l}
i \\
l
\end{array}\right) \eta^{i-l}, i \geqslant 0, \eta \in P \backslash 0 .
$$

Тогда минимальный многочлен $m_{v}(x)$ последовательности $v$ равен

$$
m_{v}(x)=(x-\eta)^{l+1} .
$$

Последовательность $v$ называется биномиальной последовательностью порядка $l$ с корнем $\eta$.

Лемма 2.6 ([11], [8], [9], [14]). Пусть $F(x)$ - многочлен Галуа над кольцом Галуа $R=G R\left(q^{n}, p^{n}\right), p \geqslant 3, m=\operatorname{deg} F(x)$, и пусть $u-Л Р \Pi$ над $R$ с характеристическим многочленом $F(x)$. Обозначим через $\theta$ корень многочлена $F(x)$ в его кольце разложения $S=G R\left(q^{m n}, p^{n}\right)$. Тогда

$(a)$ многочлен $F(x)$ является многочленом полного периода, m.е. $\mathrm{T}(F)=$ $\mathrm{T}(\bar{F}) p^{n-1}$, в том и только в том случае, когда $\gamma_{1}(\theta) \neq 0$,

(b) если $F(x)$ - многочлен полного периода, то ЛРП и является линейной рекуррентой полного периода, т.е. $\mathrm{T}(u)=\mathrm{T}(\bar{u}) p^{n-1}$, в том и только в том случае, когда $\gamma_{0}(u) \neq 0^{\infty}$ 
Кроме выше изложенных, нам также потребуются результаты работы [17], которые, в целях независимости и полноты изложения, уместно здесь привести.

Для произвольного кольца $R$ через $L_{R}(F)$ обозначается множество линейных рекуррент с характеристическим многочленом $F(x)$. Через $L_{R}\left(F_{1}\right) \cdots L_{R}\left(F_{k}\right)$ обозначается линейная оболочка множества всех последовательностей, полученных как поточечные произведения линейных рекуррент из $L_{R}\left(F_{1}\right), \ldots, L_{R}\left(F_{k}\right)$. Из результатов [17] следует, что если $P=G F(q)$ - конечное поле, $F_{1}(x), \ldots, F_{k}(x)$ - набор унитарных многочленов над ним, то имеет место равенство

$$
L_{P}\left(F_{1}\right) \cdots L_{P}\left(F_{k}\right)=L_{P}(H)
$$

где многочлен $H(x) \in P[x]$ описывается следующим образом.

Введем обозначения. Пусть $a^{(1)}, \ldots, a^{(t)} \in \mathbb{N}$ и

$$
a^{(t)}-1=\sum_{\nu \geqslant 0} \zeta_{\nu}^{(t)} p^{\nu} \in \mathbb{N}_{0}, 0 \leqslant \zeta_{\nu}<p, t=1, \ldots, k
$$

Положим

$$
\bigvee_{t=1}^{k} a^{(t)}=p^{\lambda}+\sum_{\nu \geqslant \lambda}\left(\sum_{t=1}^{k} \zeta_{\nu}^{(t)}\right) p^{\nu}
$$

где

$$
\lambda=\min \left\{\lambda \geqslant 0: \forall \nu \geqslant \lambda \sum_{t=1}^{k} \zeta_{\nu}^{(t)}<p\right\}
$$

Кроме того, для многочленов $F(x), G(x), \ldots, U(x) \in P[x]$ через

$$
F(x) \vee G(x) \vee \cdots \vee U(x)
$$

обозначим многочлен, корнями которого являются все различные произведения корней многочленов $F(x), G(x), \ldots, U(x)$ в их общем поле разложения. При этом $F(x) \vee G(x) \vee \cdots \vee U(x) \in P[x]$.

Пусть

$$
F_{s}(x)=\prod_{j_{s}=1}^{l_{s}} G_{j_{s}}^{(s)}(x)^{b_{j_{s}}^{(s)}}, s=1, \ldots, k
$$

- каноническое разложение многочленов.

Тогда

$$
H(x)=\operatorname{HOK}\left\{\left(\vee_{s=1}^{k} G_{j_{s}}^{(s)}(x)\right)^{\stackrel{k}{k} b_{j}^{(s)} b_{s}}: j_{s} \in\left\{1, \ldots, l_{s}\right\}, s=1, \ldots, k\right\} .
$$

Теперь мы можем приступить к рассмотрению последовательности $u_{2}=\gamma_{2}(u)$. 


\section{3. Основные результаты}

Напомним, что элемент $\nabla$ был определен следующим образом: согласно теореме Вильсона $(p-1) ! \underset{p}{\bar{p}}-1$. Следовательно, при $p \geqslant 3$ число $(p-1)$ ! как элемент кольца $\mathbb{Z}_{p^{n}}$ имеет представление вида

$$
(p-1) ! \underset{p^{2}}{\equiv}-1-p \nabla, \nabla \in \Gamma\left(\mathbb{Z}_{p^{n}}\right) .
$$

Кроме того, через $\theta_{0}$ обозначим $\gamma_{0}(\theta)$, где $\theta$ - корень многочлена $F(x)$ в расширении Галуа $S$ кольца $R$.

Введем обозначения:

$$
\begin{gathered}
G(x)=\prod_{\vec{\jmath} \in \Xi}\left(x \ominus \theta_{0}^{\sum_{l=0}^{m-1} j_{l} p^{r m+r l-1}}\right), \\
\Xi=\left\{\vec{\jmath} \in \mathcal{I}(m, p): \nabla \cdot \gamma_{0}\left(\frac{1}{\prod_{l=0}^{m-1} j_{l} !}\right) \neq \ominus \gamma_{1}\left(\frac{1}{\prod_{l=0}^{m-1} j_{l} !}\right)\right\}, \\
W(x)=\prod_{s=0}^{p} \prod_{(\vec{\lambda}, \vec{\zeta}) \in \Omega_{s}}\left(x \ominus \theta_{0}^{\sum_{l=0}^{m-1} p^{t+r l-2}\left(\lambda_{l}+p \zeta_{l}\right)}\right),
\end{gathered}
$$

где

$$
\begin{gathered}
\Omega_{s}=\{(\vec{\lambda}, \vec{\zeta}): \vec{\lambda} \in \mathcal{I}(m, p s), \vec{\zeta} \in \mathcal{I}(m, p-s), \\
\left.\bigoplus_{\substack{\vec{u} \in \mathcal{I}(\mathcal{N}, p): \\
\sum_{i=1}^{\mathcal{N}} \mu_{i} \cdot j_{l}^{(i)}=\lambda_{l}+p \zeta_{l}, \forall l \in \overline{0, m-1}}} \gamma_{0}\left(\frac{1}{\prod_{i=1}^{\mathcal{N}} \mu_{i} !\left(\prod_{l=0}^{m-1} j_{l}^{(i)} !\right)^{\mu_{i}}}\right) \neq 0\right\}, s=0, \ldots, p, \\
H_{s}(x)=\prod_{\substack{\vec{\lambda} \in \mathcal{I}(m, p s), \vec{\zeta} \in \mathcal{I}(m, p-s)}}\left(x \ominus \theta_{0}^{\sum_{l=0}^{m-1} p^{r m+r l-2}\left(\lambda_{l}+p \zeta_{l}\right)}\right), s=0, \ldots, p-1, \\
H(x)=H_{1}(x), Z(x)=H_{0}(x), \\
D_{1}(x)=\operatorname{НОД}(G(x), W(x)), D_{2}(x)=\text { НОД }(W(x), H(x)) .
\end{gathered}
$$

Отметим, что все введенные многочлены - над полем $\Gamma(S)$ и что $G(x) \mid Z(x)$.

Кроме того, пусть $\bar{F}(x)=\gamma_{0}(F(x)) \in(\Gamma(R), \oplus, \cdot)$ - многочлен, полученный взятием нулевого $p$-адического разряда каждого из коэффициентов.

Теорема 3.1. Пусть $R=G R\left(q^{n}, p^{n}\right)$ - кольц, Галуа, $q=p^{r}, p \geqslant 5, r \geqslant 2, F(x)$ - многочлен Галуа степени $m$ над кольчом $R$, ненулевал по модулю $p R$ последовательность $и \in L_{R}(F)$ представлена с помощью функиии след:

$$
u(i)=\operatorname{Tr}_{R}^{S}\left(\xi \theta^{i}\right),
$$

где $S=G R\left(q^{m n}, p^{n}\right)$ - кольцо разложения многочлена $F(x), \theta$ - коренъ $F(x)$ в $S$, константа $\xi \in S$ однозначно определена рекуррентой $u$. 
Пусть, далее, $Q=q^{m}=p^{t}, t=r m, \theta_{s}=\gamma_{s}(\theta), \xi_{s}=\gamma_{s}(\xi), s=0, \ldots, n-1$.

Тогда, если $F(x)$ - многочлен максимального периода, т.е. $\theta_{0}$ - примитивныи элемент поля $\Gamma(S)=G F(Q), u \theta_{1} \neq 0$, то для минимального многочлена второй координатной последовательности $u_{2}$ ЛРП и в р-адическом координатном множестве выполнены соотношения

$$
\begin{gathered}
\bar{F}(x)^{p+1} \cdot \frac{G(x)}{D_{1}(X)} \cdot \frac{W(x)}{D_{1}(x) \cdot D_{2}(x)} \cdot H(x)^{p} \mid m_{2}(x), \\
m_{2}(x) \mid \bar{F}(x)^{p+1} \cdot Z(x)^{\epsilon_{2}} \cdot H(x)^{p} \cdot \operatorname{HOK}\left(\frac{W(x)}{D_{2}(x)}, \prod_{s=2}^{p-1} H_{s}(x)^{\beta_{s}}\right) .
\end{gathered}
$$

При этом выполнены неравенства

$$
\epsilon_{2} \leqslant p, \beta_{s} \leqslant p-1, s=2, \ldots, p-1 .
$$

Кроме того, в перечисленных ниже случаях можно указать точные значения параметра $\epsilon_{2}$ :

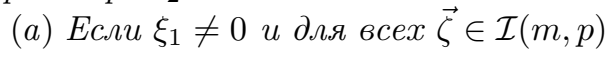

$$
\bigoplus_{\kappa=0, \ldots, m-1: \zeta_{\kappa}>0}\left(\xi_{0}^{-1} \xi_{1}\right)^{p^{t+r \kappa-1}} \neq 0
$$

$u$

$$
\bigoplus_{l=0, \ldots, m-1: \zeta_{l}>0} \gamma_{0}\left(\frac{\zeta_{l}}{\prod_{\kappa=0}^{m-1} \zeta_{\kappa} !}\right)\left(\theta_{0}^{-1} \theta_{1}\right)^{\sum_{\kappa=0}^{m-1} \zeta_{\kappa} p^{t+r \kappa-1}-p^{t+r l-1}} \neq 0,
$$

то $\epsilon_{2}=p$ и при этих же условиях $Z(x)^{p} \mid m_{2}(x)$.

(b) Если $\xi_{1}=0$, mо $\epsilon_{2}=2$. При этом $Z(x)^{2} \mid m_{2}(x)$.

Замечание 3.1. В данный момент автору неизвестны ни точное значение параметpa $\operatorname{deg} G(x)$, ни его оценка снизу. Ясно только, что этот параметр не превосходит $\left\{\begin{array}{c}m \\ p\end{array}\right\}$ и в значительной мере зависит от самого числа $p$.

Аналогично дело обстоит с параметрами $\operatorname{deg} W(x)$ и $\operatorname{deg} D_{i}(x), i=1,2$.

Замечание 3.2. Рассуждения, проводимые далее при доказательстве теоремы 3.1, справедливы также и при $R=\mathbb{Z}_{p^{n}}$. Однако при $r=1$ введенные выше обозначения становятся некорректными, и по этой причине неверными становятся оценки рангов в утверждении 3.2 .

Доказательство теоремы 3.1. Итак, пусть $R=G R\left(q^{n}, p^{n}\right)$ - собственное кольцо Галуа, $F(x) \in R[x]-$ многочлен Галуа, $u \in L_{R}(F)-$ ненулевая по модулю $p R$ линейная рекуррента с характеристическим многочленом $F(x)$. Используя формулы $(1.1),(1.3)$, легко получить, что при всех $i \in \mathbb{N}_{0}$ имеет место сравнение

$$
u(i) \underset{p^{3}}{\sum_{l=0}^{m-1}}\left(\gamma_{0}(\xi)^{q^{l}}+p \gamma_{1}(\xi)^{q^{l}}+p^{2} \gamma_{2}(\xi)^{q^{l}}\right) \cdot\left(\gamma_{0}(\theta)^{q^{l}}+p \gamma_{1}(\theta)^{q^{l}}+p^{2} \gamma_{2}(\theta)^{q^{l}}\right)^{i}
$$

Напомним, что $\xi_{s}=\gamma_{s}(\xi), \theta_{s}=\gamma_{s}(\theta), s=0, \ldots, n-1$. Тогда, раскрывая скобки и приводя подобные, равенство (3.1) можно продолжить следующим образом: 


$$
\begin{aligned}
& u(i) \underset{p^{3}}{\sum_{l=0}^{m-1}}\left(\xi_{0}^{q^{l}}+p \xi_{1}^{q^{l}}+p^{2} \xi_{2}^{q^{l}}\right) \\
& \cdot\left(\theta_{0}^{i q^{l}}+p i\left(\theta_{0}^{i-1} \theta_{1}\right)^{q^{l}}+p^{2} i\left(\theta_{0}^{i-1} \theta_{2}\right)^{q^{l}}+p^{2} \cdot \frac{i(i-1)}{2} \cdot\left(\theta_{0}^{i-2} \theta_{1}^{2}\right)^{q^{l}}\right) \overline{\overline{p^{3}}} \\
& \equiv \sum_{p^{3}}^{m-1}\left(\xi_{0} \theta_{0}^{i}\right)^{q^{l}}+p \sum_{l=0}^{m-1}\left(\left(\xi_{0}^{-1} \xi_{1}\right)^{q^{l}}+\gamma_{0}(i)\left(\theta_{0}^{-1} \theta_{1}\right)^{q^{l}}\right)\left(\xi_{0} \theta_{0}^{i}\right)^{q^{l}}+ \\
& +p^{2} \sum_{l=0}^{m-1}\left(\left(\begin{array}{l}
i \\
2
\end{array}\right)\left(\theta_{0}^{-2} \theta_{1}^{2}\right)^{q^{l}}+i\left(\left(\theta_{0}^{-1} \theta_{1} \xi_{0}^{-1} \xi_{1}\right)^{q^{l}}+\left(\theta_{0}^{-1} \theta_{2}\right)^{q^{l}}\right)+\left(\xi_{0}^{-1} \xi_{2}\right)^{q^{l}}+\gamma_{1}(i)\left(\theta_{0}^{-1} \theta_{1}\right)^{q^{l}}\right) . \\
& \cdot\left(\xi_{0} \theta_{0}^{i}\right)^{q^{l}}
\end{aligned}
$$

Введем в рассмотрение последовательности $a, b, c$, определив их следующим образом: для каждого $i \in \mathbb{N}_{0}$

$$
\begin{gathered}
a(i)=\sum_{l=0}^{m-1}\left(\left(\begin{array}{c}
i \\
2
\end{array}\right)\left(\theta_{0}^{-2} \theta_{1}^{2}\right)^{q^{l}}+i\left(\left(\theta_{0}^{-1} \theta_{1} \xi_{0}^{-1} \xi_{1}\right)^{q^{l}}+\right.\right. \\
\left.\left.+\left(\theta_{0}^{-1} \theta_{2}\right)^{q^{l}}\right)+\left(\xi_{0}^{-1} \xi_{2}\right)^{q^{l}}+\gamma_{1}(i)\left(\theta_{0}^{-1} \theta_{1}\right)^{q^{l}}\right)\left(\xi_{0} \theta_{0}^{i}\right)^{q^{l}} \\
b(i)=\sum_{l=0}^{m-1}\left(\left(\xi_{0}^{-1} \xi_{1}\right)^{q^{l}}+\gamma_{0}(i)\left(\theta_{0}^{-1} \theta_{1}\right)^{q^{l}}\right)\left(\xi_{0} \theta_{0}^{i}\right)^{q^{l}} \\
c(i)=\sum_{l=0}^{m-1}\left(\xi_{0} \theta_{0}^{i}\right)^{q^{l}} .
\end{gathered}
$$

Поскольку

$$
\begin{gathered}
u \underset{p^{3}}{\equiv} \gamma_{0}(c)+p\left(b+\gamma_{1}(c)\right)+p^{2}\left(a+\gamma_{2}(c)\right)= \\
=\gamma_{0}(c)+p \gamma_{0}\left(b+\gamma_{1}(c)\right)+p^{2}\left(a+\gamma_{2}(c)+\gamma_{1}\left(b+\gamma_{1}(c)\right)\right),
\end{gathered}
$$

имеет место равенство

$$
u_{2}=\gamma_{0}(a) \oplus \gamma_{2}(c) \oplus \gamma_{1}\left(b+\gamma_{1}(c)\right)
$$

Из (3.6) следует, что

$$
m_{2}(x) \mid \operatorname{HOK}\left[m_{\gamma_{0}(a)}(x), m_{\gamma_{2}(c)}(x), m_{\gamma_{1}\left(b+\gamma_{1}(c)\right)}(x)\right] .
$$

Кроме того, из (3.6) следует, что многочлен $m_{2}(x)$ делится на каждый из примарных многочленов $U^{\epsilon}(x)$, входящих в каноническое разложение какого-либо из многочленов $m_{\gamma_{0}(a)}(x), m_{\gamma_{2}(c)}(x), m_{\gamma_{1}\left(b+\gamma_{1}(c)\right)}(x)$, по крайней мере, в следующих двух случаях:

либо $\epsilon$ имеет максимальное значение и многочлен $U(x)$ не входит в каноническое разложение минимальных многочленов двух других рассматриваемых последовательностей в той же степени $\epsilon$, 
либо $\epsilon=1$ и

$$
\begin{gathered}
\frac{m_{\gamma_{0}(a)}(x)}{\text { НОД }\left(m_{\gamma_{0}(a)}(x), U(x)\right)} \cdot \gamma_{0}(a) \oplus \frac{m_{\gamma_{2}(c)}(x)}{\text { НОД }\left(m_{\gamma_{2}(c)}(x), U(x)\right)} \cdot \gamma_{2}(c) \oplus \\
\oplus \frac{m_{\gamma_{1}\left(b+\gamma_{1}(c)\right)}(x)}{\text { НОД }\left(m_{\gamma_{1}\left(b+\gamma_{1}(c)\right)}(x), U(x)\right)} \cdot \gamma_{1}\left(b+\gamma_{1}(c)\right) \neq 0 .
\end{gathered}
$$

Таким образом, для решения нашей задачи следует найти минимальные многочлены последовательностей, фигурирующих в правой части равенства (3.6).

Используем обозначения, введённые в начале раздела 3.

Каждый из полиномов $G(x), W(x), H_{s}(x)$ лежит в кольце $\Gamma(S)[x]$. Более того, поскольку многочлены $G(x), H_{s}(x), s=0, \ldots, p-1, W(x)$ при возведении в степень $q$ дают, соответственно, $G\left(x^{q}\right), H_{s}\left(x^{q}\right), s=0, \ldots, p-1, W\left(x^{q}\right)$, то каждый из них лежит в кольце многочленов над полем $\Gamma(R)$.

Кроме того, отметим, что $G(x) \mid Z(x)$.

Напомним, что по условию $\theta_{0} \neq 0, \xi_{0} \neq 0$.

Лемма 3.1. Имеет место равенство

$$
m_{\gamma_{0}(a)}(x)=\bar{F}(x)^{\epsilon_{a}},
$$

¿əe

$$
\epsilon_{a}= \begin{cases}p+1, & \theta_{1} \neq 0 \\ 2, & \theta_{1}=0, \theta_{2} \neq 0 \\ 1, & \theta_{1}=\theta_{2}=0, \xi_{2} \neq 0 \\ 0, & \theta_{1}=\theta_{2}=\xi_{2}=0 .\end{cases}
$$

Доказательство. Разберем последовательно указанные случаи. Пусть сначала $\theta_{1} \neq 0$. Тогда

$$
\begin{array}{r}
\gamma_{0}(a)(i)=\bigoplus_{l=0}^{m-1}\left(\left(\begin{array}{l}
i \\
2
\end{array}\right)\left(\theta_{0}^{-2} \theta_{1}^{2}\right)^{q^{l}} \oplus i\left(\left(\theta_{0}^{-1} \theta_{1} \xi_{0}^{-1} \xi_{1}\right)^{q^{l}} \oplus\left(\theta_{0}^{-1} \theta_{2}\right)^{q^{l}}\right) \oplus\left(\xi_{0}^{-1} \xi_{2}\right)^{q^{l}}\right)\left(\xi_{0} \theta_{0}^{i}\right)^{q^{l}} \oplus \\
\oplus \bigoplus_{l=0}^{m-1} \gamma_{1}(i)\left(\theta_{0}^{-1} \theta_{1}\right)^{q^{l}}\left(\xi_{0} \theta_{0}^{i}\right)^{q^{l}} . \quad(3.10)
\end{array}
$$

Обозначим первую сумму в правой части равенства (3.10) через $a_{1}(i)$, вторую сумму обозначим через $a_{2}(i)$. В соответствии со свойствами биномиальных последовательностей имеет место равенство

$$
m_{a_{1}}(x)=\bar{F}(x)^{3},
$$

и согласно [17]

$$
m_{a_{2}}(x)=(x \ominus e)^{p+1} \vee \bar{F}(x)=\bar{F}(x)^{p+1} .
$$

Отсюда и из условия $p \geqslant 5$ следует, что $m_{\gamma_{0}(a)}(x)=m_{a_{2}}(x)=\bar{F}(x)^{p+1}$.

Пусть теперь $\theta_{1}=0$. Тогда

$$
\gamma_{0}(a)(i)=\bigoplus_{l=0}^{m-1}\left(i\left(\theta_{0}^{-1} \theta_{2}\right)^{q^{l}} \oplus\left(\xi_{0}^{-1} \xi_{2}\right)^{q^{l}}\right)\left(\xi_{0} \theta_{0}^{i}\right)^{q^{l}}
$$


Поэтому в соответствии со свойствами биномиальных последовательностей

$$
m_{\gamma_{0}(a)}(x)=\bar{F}(x)^{2} \text {, если } \theta_{2} \neq 0,
$$

и

$$
m_{\gamma_{0}(a)}(x)=\bar{F}(x), \text { если } \theta_{2}=0, \xi_{2} \neq 0 .
$$

Если же $\theta_{2}=0, \xi_{2}=0$, то $\gamma_{0}(a)=0^{\infty}$, т. е. $m_{\gamma_{0}(a)}(x)=e$.

Найдем теперь $m_{\gamma_{2}(c)}(x)$.

Лемма 3.2. Если $\theta_{0}$ - примитивный элемент поля $\Gamma(S)$, то имеют место соотношения

$$
\frac{G(x)}{D_{1}(x)} \cdot \frac{W(x)}{D_{1}(x) \cdot D_{2}(x)}\left|m_{\gamma_{2}(c)}(x)\right| \frac{G(x)}{D_{1}(x)} \cdot \frac{W(x)}{D_{2}(x)} \cdot H(x) .
$$

Здесь

$$
D_{1}(x)=\text { НОД}(W(x), G(x)), D_{2}(x)=\text { НОД }(W(x), H(x)) .
$$

Доказательство. Напомним, что мы используем обозначения $q=p^{r}, t=r m$. Согласно лемме 2.3

$$
\begin{aligned}
& \gamma_{2}(c)(i)=\gamma_{2}\left(\sum_{l=0}^{m-1}\left(\xi_{0} \theta_{0}^{i}\right)^{q^{l}}\right)= \\
& =W^{(Q, 1)}\left(\xi_{0} \theta_{0}^{i}, \ldots,\left(\xi_{0} \theta_{0}^{i}\right)^{q^{m-1}}\right) \oplus W^{(Q, 2,1)}\left(\xi_{0} \theta_{0}^{i}, \ldots,\left(\xi_{0} \theta_{0}^{i}\right)^{q^{m-1}}\right) \ominus \\
& \ominus W^{(Q, 2,2)}\left(\xi_{0} \theta_{0}^{i}, \ldots,\left(\xi_{0} \theta_{0}^{i}\right)^{q^{m-1}}\right)= \\
& =\bigoplus_{\vec{\jmath} \in \mathcal{I}(m, p)} w_{\vec{\jmath}}^{(Q, 1)} \prod_{l=0}^{m-1}\left(\xi_{0} \theta_{0}^{i}\right)^{q^{l} \cdot j_{l} \cdot p^{t-1}} \oplus \bigoplus_{s=0}^{p} \bigoplus_{\substack{\vec{\lambda} \in \mathcal{I}(m, p s), \vec{\zeta} \in \mathcal{I}(m, p-s)}} w_{\vec{\lambda}, \vec{\zeta}}^{(Q, 2,1)} \prod_{l=0}^{m-1}\left(\xi_{0} \theta_{0}^{i}\right)^{q^{l} \cdot p^{t-2} \cdot\left(\lambda_{l}+p \zeta_{l}\right)} \ominus \\
& \ominus \bigoplus_{\substack{\vec{\lambda} \in \mathcal{I}(m, p), \vec{\zeta} \in \mathcal{I}(m, p-1)}} w_{\vec{\lambda}, \vec{\zeta}}^{(Q, 2,2)} \prod_{l=0}^{m-1}\left(\xi_{0} \theta_{0}^{i}\right)^{q^{l} \cdot p^{t-2} \cdot\left(\lambda_{l}+p \zeta_{l}\right)}= \\
& =\bigoplus_{\vec{\jmath} \in \mathcal{I}(m, p)} w_{\vec{\jmath}}^{(Q, 1)} \xi_{0}^{\sum_{l=0}^{m-1} j_{l} \cdot p^{t+r l-1}} \cdot\left(\theta_{0}^{\sum_{l=0}^{m-1} j_{l} \cdot p^{t+r l-1}}\right)^{i} \oplus \\
& \oplus \bigoplus_{s=0}^{p} \bigoplus_{\substack{\lambda \in \mathcal{I}(m, p s), \vec{\zeta} \in \mathcal{I}(m, p-s)}} w_{\vec{\lambda}, \vec{\zeta}}^{(Q, 2,1)} \xi_{0}^{\sum_{l=0}^{m-1} p^{t+r l-2} \cdot\left(\lambda_{l}+p \zeta_{l}\right)} \cdot\left(\theta_{0}^{\sum_{l=0}^{m-1} p^{t+r l-2} \cdot\left(\lambda_{l}+p \zeta_{l}\right)}\right)^{i} \ominus \\
& \ominus \bigoplus_{\vec{\lambda} \in \mathcal{I}(m, p),} w_{\vec{\lambda}, \vec{\zeta}}^{(Q, 2,2)} \xi_{0}^{\sum_{l=0}^{m-1} p^{t+r l-2} \cdot\left(\lambda_{l}+p \zeta_{l}\right)} \cdot\left(\theta_{0}^{\sum_{l=0}^{m-1} p^{t+r l-2} \cdot\left(\lambda_{l}+p \zeta_{l}\right)}\right)^{i} . \\
& \vec{\zeta} \in \mathcal{I}(m, p-1)
\end{aligned}
$$

Корни выписанных выше биномиальных последовательностей попарно различны в силу примитивности элемента $\theta_{0}$, за исключением следующих двух случаев:

1) корни биномиальных последовательностей, входящих во вторую сумму при $s=0$, и последовательностей, входящих в первую сумму, могут совпадать,

2) корни биномиальных последовательностей, входящих во вторую сумму при $s=1$, и последовательностей, входящих в третью сумму, также могут совпадать.

Кроме того, $w_{\vec{\lambda}, \vec{\zeta}}^{(Q, 2,2)} \neq 0$ при всех $\vec{\lambda} \in \mathcal{I}(m, p), \vec{\zeta} \in \mathcal{I}(m, p-1)$.

Из предыдущего получаем утверждение леммы.

Обозначим $\tilde{b}=\gamma_{1}\left(b+\gamma_{1}(c)\right)$. Теперь для нахождения многочлена $m_{2}(x)$ остается найти $m_{\tilde{b}}(x)$. 
Лемма 3.3. В условиях леммы 3.2 имеют место соотношения

$$
H(x)^{\epsilon_{b}^{(1)}} \cdot \bar{F}(x)^{\epsilon_{b}^{(3)}}\left|m_{\tilde{b}}(x)\right| H(x)^{\epsilon_{b}^{(1)}} \cdot Z(x)^{\epsilon_{b}^{(2)}} \cdot \bar{F}(x)^{\epsilon_{b}^{(3)}} \cdot \prod_{s=2}^{p-1} H_{s}(x)^{\beta_{s}},
$$

где $\epsilon_{b}^{(1)} \leqslant p, \epsilon_{b}^{(2)} \leqslant p, \beta_{s} \leqslant p-1, s=2, \ldots, p-1$, и в следующих случалх можно указать точные значения параметров $\epsilon_{b}^{(1)}, \epsilon_{b}^{(2)} u \epsilon_{b}^{(3)}$ :

(а) Если $\theta_{1} \neq 0, \xi_{1} \neq 0$, то $\epsilon_{b}^{(1)}=p, \epsilon_{b}^{(3)}=p$, и если дополнителъно

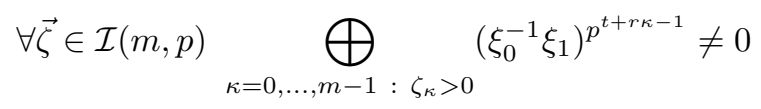

$u$

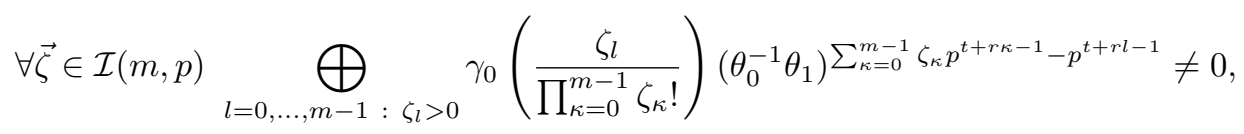

mо $\epsilon_{b}^{(2)}=p$. При этих же условиях

$$
Z(x)^{\epsilon_{b}^{(2)}} \mid m_{\tilde{b}}(x) .
$$

(b) Если $\theta_{1} \neq 0, \xi_{1}=0$, mо $\epsilon_{b}^{(1)}=p, \epsilon_{b}^{(2)}=2, \epsilon_{b}^{(3)}=0$. При этих жсе условиях

$$
Z(x)^{\epsilon_{b}^{(2)}} \mid m_{\tilde{b}}(x) \text {. }
$$

Доказательство. Разберем последовательно указанные случаи. Пусть сначала $\theta_{1} \neq 0, \xi_{1} \neq 0$. Тогда с учетом $(2.38)$ имеем:

$$
\begin{aligned}
& \tilde{b}(i)=\gamma_{1}\left(\sum_{l=0}^{m-1}\left(\left(\xi_{0}^{-1} \xi_{1}\right)^{q^{l}}+\gamma_{0}(i)\left(\theta_{0}^{-1} \theta_{1}\right)^{q^{l}}\right)\left(\xi_{0} \theta_{0}^{i}\right)^{q^{l}}+\gamma_{1}(c)\right)=
\end{aligned}
$$

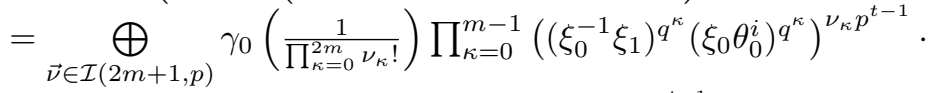

$$
\begin{aligned}
& \cdot \prod_{\kappa=m}^{2 m-1}\left(\gamma_{0}(i)\left(\theta_{0}^{-1} \theta_{1}\right)^{q^{\kappa-m}} \cdot\left(\xi_{0} \theta_{0}^{i}\right)^{q^{\kappa-m}}\right)^{\nu_{\kappa} p^{t-1}} \cdot \gamma_{1}(c)^{\nu_{2 m} p^{t-1}}= \\
& =\bigoplus_{\vec{\nu} \in \mathcal{I}(2 m+1, p)} \gamma_{0}\left(\frac{1}{\prod_{\kappa=0}^{2 m} \nu_{\kappa} !}\right) \cdot\left(\xi_{0}^{-1} \xi_{1}\right)^{\sum_{\kappa=0}^{m-1} \nu_{\kappa} p^{t+r \kappa-1}} \cdot\left(\theta_{0}^{-1} \theta_{1}\right)^{\sum_{\kappa=m}^{2 m-1} \nu_{\kappa} p^{t+r \kappa-1}} \text {. } \\
& \cdot \gamma_{0}(i)^{\sum_{\kappa=m}^{2 m-1} \nu_{\kappa}} \cdot\left(\xi_{0} \theta_{0}^{i}\right)^{\sum_{l=0}^{m-1} p^{t+r l-1}\left(\nu_{l}+\nu_{l+m}\right)} \cdot \gamma_{1}(c)^{\nu_{2 m} p^{t-1}} \text {. }
\end{aligned}
$$

Рассмотрим отдельно сомножитель $\gamma_{1}(c)^{\nu_{2 m} p^{t-1}}$.

Поскольку имеет место равенство

$$
\gamma_{1}(c)=\bigoplus_{\vec{\jmath} \in \mathcal{I}(m, p)} \gamma_{0}\left(\frac{1}{\prod_{l=0}^{m-1} j_{l} !}\right)\left(\xi_{0} \theta_{0}^{i}\right)^{\sum_{l=0}^{m-1} j_{l} p^{t+r l-1}},
$$

Tо

$$
\begin{aligned}
& \gamma_{1}(c)^{\nu_{2 m} p^{t-1}}=\left(\bigoplus_{\vec{\jmath} \in \mathcal{I}(m, p)} \gamma_{0}\left(\frac{1}{\prod_{l=0}^{m-1} j_{l} !}\right)\left(\xi_{0} \theta_{0}^{i}\right)^{\sum_{l=0}^{m-1} j_{l} p^{t+r l-2}}\right)^{\nu_{2 m}}= \\
& =\bigoplus_{\vec{\eta} \in \mathcal{I}\left(\mathcal{N}, \nu_{2 m}\right)} \gamma_{0}\left(\frac{\nu_{2 m} !}{\prod_{s=1}^{\mathcal{N}} \eta_{s} !\left(\prod_{l=0}^{m-1} j_{l}^{(s)} !\right)^{\eta_{s}}}\right) \cdot\left(\xi_{0} \theta_{0}^{i}\right)^{\sum_{l=0}^{m-1} p^{t+r l-2} \sum_{s=1}^{N} \eta_{s} j_{l}^{(s)}} .
\end{aligned}
$$


Здесь, как и ранее, мы считаем, что $\mathcal{N}=\left\{\begin{array}{c}m \\ p\end{array}\right\}$ и что векторы $\vec{\jmath} \in \mathcal{I}(m, p)$ пронумерованы в лексикографическом порядке.

Из соотношений (3.17), (3.19) следует, что

$$
\begin{aligned}
& \tilde{b}(i)=\bigoplus_{\vec{\nu} \in \mathcal{I}(2 m+1, p) \vec{\eta} \in \mathcal{I}\left(\mathcal{N}, \nu_{2 m}\right)} \gamma_{0}\left(\frac{1}{\prod_{\kappa=0}^{2 m-1} \nu_{\kappa} !} \times \frac{1}{\prod_{s=1}^{\mathcal{N}} \eta_{s} !\left(\prod_{l=0}^{m-1} j_{l}^{(s)} !\right)^{\eta_{s}}}\right) \times \\
& \times\left(\xi_{0}^{-1} \xi_{1}\right)^{\sum_{\kappa=0}^{m-1} \nu_{\kappa} p^{t+r \kappa-1}} \cdot\left(\theta_{0}^{-1} \theta_{1}\right)^{\sum_{\kappa=m}^{2 m-1} \nu_{\kappa} p^{t+r \kappa-1}} \times \\
& \times \gamma_{0}(i)^{\sum_{\kappa=m}^{2 m-1} \nu_{\kappa}} \cdot\left(\xi_{0} \theta_{0}^{i}\right)^{\sum_{l=0}^{m-1} p^{t+r l-2}\left(p\left(\nu_{l}+\nu_{l+m}\right)+\sum_{s=1}^{\mathcal{N}} \eta_{s} j_{l}^{(s)}\right)} .
\end{aligned}
$$

Рассмотрим подробнее показатель степени при $\left(\xi_{0} \theta_{0}^{i}\right)$.

При любом фиксированном значении параметра $l \in\{0, \ldots, m-1\}$ имеют место соотношения

$$
\begin{aligned}
& p\left(\nu_{l}+\nu_{l+m}\right)+\sum_{s=1}^{\mathcal{N}} \eta_{s} j_{l}^{(s)} \leqslant p\left(\nu_{l}+\nu_{l+m}\right)+(p-1) \sum_{s=1}^{\mathcal{N}} \eta_{s}= \\
& =p\left(\nu_{l}+\nu_{l+m}\right)+(p-1) \nu_{2 m} \leqslant p\left(p-\nu_{2 m}\right)+(p-1) \nu_{2 m}=p^{2}-\nu_{2 m} \leqslant p^{2} .
\end{aligned}
$$

Кроме того,

$$
\begin{aligned}
& \sum_{l=0}^{m-1}\left(p\left(\nu_{l}+\nu_{l+m}\right)+\sum_{s=1}^{\mathcal{N}} \eta_{s} j_{l}^{(s)}\right)=p\left(p-\nu_{2 m}\right)+\sum_{s=1}^{\mathcal{N}} \eta_{s} \sum_{l=0}^{m-1} j_{l}^{(s)}= \\
& =p\left(p-\nu_{2 m}\right)+p \nu_{2 m}=p^{2} .
\end{aligned}
$$

Следовательно, для каждой пары $\vec{\nu} \in \mathcal{I}(2 m+1, p), \vec{\eta} \in \mathcal{I}\left(\mathcal{N}, \nu_{2 m}\right)$ показатель степени при $\xi_{0} \theta_{0}^{i}$ можно однозначно представить в виде

$$
\sum_{l=0}^{m-1} p^{t+r l-2}\left(\lambda_{l}+p \zeta_{l}\right)
$$

причем пара $\vec{\lambda}=\left(\lambda_{0}, \ldots, \lambda_{m-1}\right), \vec{\zeta}=\left(\zeta_{1}, \ldots, \zeta_{m-1}\right)$ при $\nu_{2 m} \neq 0$ пробегает множество

$$
\bigcup_{s=1}^{p-1}(\mathcal{I}(m, p s) \times \mathcal{I}(m, p-s)),
$$

где $\times$ обозначает декартово произведение множеств.

При $\nu_{2 m}=0$ пара векторов $\vec{\lambda}, \vec{\zeta}$ пробегает множества

$$
\{\overrightarrow{0}\} \times \mathcal{I}(m, p) \text { и }\{\overrightarrow{0}\} \times \bigcup_{l=0}^{m-1} p \cdot \vec{e}_{l},
$$

где $\vec{e}_{l}-l$-я строка единичной $m \times m$-матрицы.

Последнее множество входит в область суммирования тогда и только тогда, когда

$$
\nu_{l}+\nu_{l+m}=p, \nu_{\kappa}=0, \kappa \neq l, l+m, l=0, \ldots, m-1
$$


Таким образом, равенство (3.20) можно продолжить следующим образом:

$$
\begin{aligned}
& \tilde{b}(i)=\bigoplus_{s=1}^{p-1} \bigoplus_{\substack{\vec{\lambda} \in \mathcal{I}(m, p s) \\
\vec{\zeta} \in \mathcal{I}(m, p-s)}}\left(\xi_{0} \theta_{0}^{i}\right)^{\sum_{l=0}^{m-1} p^{t+r l-2}\left(\lambda_{l}+p \zeta_{l}\right)} \times \\
& \times\left(\bigoplus_{\Psi(\tilde{b})} \gamma_{0}\left(\frac{1}{\prod_{\kappa=0}^{2 m-1} \nu_{\kappa} !} \cdot \frac{1}{\prod_{s=1}^{\mathcal{N}} \eta_{s} !\left(\prod_{l=0}^{m-1} j_{l}^{(s)} !\right)^{\eta_{s}}}\right) \times\right. \\
& \left.\times\left(\xi_{0}^{-1} \xi_{1}\right)^{\sum_{\kappa=0}^{m-1} \nu_{\kappa} p^{t+r \kappa-1}} \cdot\left(\theta_{0}^{-1} \theta_{1}\right)^{\sum_{\kappa=m}^{2 m-1} \nu_{\kappa} p^{t+r \kappa-1}} \cdot \gamma_{0}(i)^{\sum_{\kappa=m}^{2 m-1} \nu_{\kappa}}\right) \oplus \\
& \oplus \bigoplus_{\vec{\zeta} \in \mathcal{I}(m, p)}\left(\xi_{0} \theta_{0}^{i}\right)^{\sum_{l=0}^{m-1} p^{t+r l-1} \zeta_{l}} \times\left(\sum_{\substack{\vec{\nu} \in \mathcal{I}(2 m, p): \\
\nu_{l}+\nu_{l+m}=\zeta_{l}}} \oplus \gamma_{0}\left(\frac{1}{\prod_{\kappa=0}^{2 m-1} \nu_{\kappa} !}\right) .\right. \\
& \left.\cdot\left(\xi_{0}^{-1} \xi_{1}\right)^{\sum_{\kappa=0}^{m-1} \nu_{\kappa} p^{t+r \kappa-1}} \cdot\left(\theta_{0}^{-1} \theta_{1}\right) \sum_{\kappa=m}^{2 m-1} \nu_{\kappa} p^{t+\kappa \kappa-1} \cdot \gamma_{0}(i) \sum_{\kappa=m}^{2 m-1} \nu_{\kappa}\right) \oplus \\
& \oplus \bigoplus_{l=0}^{m-1} \bigoplus_{\vec{\nu} \in \mathcal{I}(2, p)} \gamma_{0}\left(\frac{1}{\nu_{0} ! \nu_{1} !}\right) \cdot\left(\xi_{0}^{-1} \xi_{1}\right)^{\nu_{0} p^{t+r l-1}} \cdot\left(\theta_{0}^{-1} \theta_{1}\right)^{\nu_{1} p^{t+r l-1}} \cdot \gamma_{0}(i)^{\nu_{1}} \cdot\left(\xi_{0} \theta_{0}^{i}\right)^{p^{t+r l}},
\end{aligned}
$$

где

$$
\begin{aligned}
& \Psi(\tilde{b})=\left\{\vec{\nu} \in \mathcal{I}(2 m+1, p), \vec{\eta} \in \mathcal{I}\left(\mathcal{N}, \nu_{2 m}\right):\right. \\
& \left.\nu_{2 m} \neq 0, p\left(\nu_{l}+\nu_{l+m}\right)+\sum_{s=1}^{\mathcal{N}} \eta_{s} j_{l}^{(s)}=\lambda_{l}+p \zeta_{l} \forall l \in\{0, \ldots, m-1\}\right\} .
\end{aligned}
$$

Таким образом, мы представили последовательность $\tilde{b}$ в виде суммы биномиальных (полиномиальных) последовательностей с попарно различными корнями.

Рассмотрим сначала минимальный многочлен последовательности, представленной третьей суммой в равенстве (3.24). Указанную формулу можно продолжить следующим образом:

$$
\begin{aligned}
& \bigoplus_{l=0}^{m-1} \bigoplus_{\vec{\nu} \in \mathcal{I}(2, p)} \gamma_{0}\left(\frac{1}{\nu_{0} ! \nu_{1} !}\right) \cdot\left(\xi_{0}^{-1} \xi_{1}\right)^{\nu_{0} p^{t+r l-1}} \cdot\left(\theta_{0}^{-1} \theta_{1}\right)^{\nu_{1} p^{t+r l-1}} \cdot \gamma_{0}(i)^{\nu_{1}} \cdot\left(\xi_{0} \theta_{0}^{i}\right)^{p^{t+r l}}= \\
& =\bigoplus_{l=0}^{m-1}\left(\xi_{0} \theta_{0}^{i}\right)^{p^{r l}} \times \\
& \left.\times \bigoplus_{\vec{\nu} \in \mathcal{I}(2, p)} \gamma_{0}\left(\frac{1}{\nu_{0} ! \nu_{1} !}\right) \cdot\left(\left(\xi_{0}^{-1} \xi_{1}\right)^{p^{t+r l-1}}\right)^{\nu_{0}} \cdot\left(\left(\theta_{0}^{-1} \theta_{1}\right)^{p^{t+r l-1}} \cdot \gamma_{0}(i)\right)^{\nu_{1}}\right)= \\
& =\bigoplus_{l=0}^{m-1}\left(\xi_{0} \theta_{0}^{i}\right)^{p^{r l}} \cdot \gamma_{1}\left(\left(\xi_{0}^{-1} \xi_{1}\right)^{p^{t+r l-1}}+\gamma_{0}(i) \cdot\left(\theta_{0}^{-1} \theta_{1}\right)^{p^{t+r l-1}}\right) .
\end{aligned}
$$

Заметим, что выписанная последовательность есть сумма последовательностей вида

$$
\left(\xi_{0} \theta_{0}^{i}\right)^{q^{l}} \cdot \gamma_{0}(i)^{\nu_{1}}, \quad l=0, \ldots, m-1, \nu_{1}=1, \ldots, p-1
$$


с ненулевыми коэффициентами. Следовательно, минимальный многочлен этой последовательности есть $\bar{F}(x)^{\beta}$, где $\beta$ определяется максимальным показателем степени, в которой в выписанные подпоследовательности входит в качестве сомножителя выражение $\gamma_{0}(i)$. Выписанная последовательность содержит в качестве подпоследовательности последовательность вида

$$
\ominus \bigoplus_{l=0}^{m-1}\left(\xi_{0}^{-1} \xi_{1}\right)^{p^{t+r l-1}}\left(\theta_{0}^{-1} \theta_{1}\right)^{(p-1) p^{t+r l-1}} \gamma_{0}(i)^{p-1}\left(\xi_{0} \theta_{0}^{i}\right)^{q^{l}}
$$

полученную путем группирования всех членов последовательности (3.25) при $\nu_{1}=$ $p-1$. Минимальный многочлен последовательности (3.26) равен

$$
\bar{F}(x) \vee \underbrace{(x \ominus e)^{2} \vee \cdots \vee(x \ominus e)^{2}}_{\text {дизъюнкция } p-1 \text { сомножителей }}=\bar{F}(x) \underbrace{2 \vee \cdots \vee 2}_{p-1}=\bar{F}(x)^{p} .
$$

Следовательно, минимальный многочлен последовательности (3.25) равен

$$
\tilde{F}(x)^{p}
$$

Рассмотрим теперь минимальный многочлен последовательности, представленной второй суммой в равенстве (3.24). Этот минимальный многочлен есть степень многочлена $Z(x)$, причем показатель степени определяется максимальным значением показателя степени при $\gamma_{0}(i)$, с которым это выражение в качестве сомножителя входит в суммируемые подпоследовательности, составляющие указанную последовательность .

Максимальное значение показателя степени при $\gamma_{0}(i)$ равно $p-1$ и достигается при

$$
\sum_{\kappa=m}^{2 m-1} \nu_{\kappa}=p-1
$$

Сгруппируем слагаемые, отвечающие этому условию, и выпишем получающуюся последовательность:

$$
\begin{aligned}
& \bigoplus\left(\xi_{0} \theta_{0}^{i}\right)^{\sum_{l=0}^{m-1} p^{t+r l-1} \zeta_{l}} \times \gamma_{0}(i)^{p-1} \times \\
& \vec{\zeta} \in \mathcal{I}(m, p) \\
& \times\left(\sum_{\substack{\vec{\nu} \in \mathcal{I}(2 m, p): \nu_{l}+\nu_{l+m}=\zeta_{l} \\
\sum_{\kappa=m}^{2 m-1} \nu_{\kappa}=p-1}} \gamma_{0}\left(\frac{1}{\prod_{\kappa=0}^{2 m-1} \nu_{\kappa} !}\right)\left(\xi_{0}^{-1} \xi_{1}\right)^{\sum_{\kappa=0}^{m-1} \nu_{\kappa} p^{t+r \kappa-1}}\left(\theta_{0}^{-1} \theta_{1}\right)^{\sum_{\kappa=m}^{2 m-1} \nu_{\kappa} p^{t+r \kappa-1}}\right)= \\
& =\bigoplus_{\vec{\zeta} \in \mathcal{I}(m, p)}\left(\xi_{0} \theta_{0}^{i}\right)^{\sum_{l=0}^{m-1} p^{t+r l-1} \zeta_{l}} \times \gamma_{0}(i)^{p-1} \times
\end{aligned}
$$




$$
\begin{aligned}
& \times\left(\bigoplus_{\kappa=0, \ldots, m-1: \zeta_{\kappa}>0}\left(\xi_{0}^{-1} \xi_{1}\right)^{p^{t+r \kappa-1}}\right. \\
& \left.\bigoplus_{\substack{l=0, \ldots, m-1: \zeta_{l}>0 ; \\
\vec{\nu} \in \mathcal{I}(m, p-1): \vec{\nu}+\vec{e}_{l}=\vec{\zeta}}} \gamma_{0}\left(\frac{1}{\prod_{\kappa=0}^{m-1} \nu_{\kappa} !}\right)\left(\theta_{0}^{-1} \theta_{1}\right)^{\sum_{\kappa=0}^{m-1} \nu_{\kappa} p^{t+r \kappa-1}}\right)= \\
& =\bigoplus_{\vec{\zeta} \in \mathcal{I}(m, p)}\left(\xi_{0} \theta_{0}^{i}\right)^{\sum_{l=0}^{m-1} p^{t+r l-1} \zeta_{l}} \times \gamma_{0}(i)^{p-1}\left(\bigoplus_{\kappa=0, \ldots, m-1: \zeta_{\kappa}>0}\left(\xi_{0}^{-1} \xi_{1}\right)^{p^{t+r \kappa-1}}\right) \times \\
& \times\left(\bigoplus_{l=0, \ldots, m-1: \zeta_{l}>0} \gamma_{0}\left(\frac{\zeta_{l}}{\prod_{\kappa=0}^{m-1} \zeta_{\kappa} !}\right)\left(\theta_{0}^{-1} \theta_{1}\right)^{\sum_{\kappa=0}^{m-1} \zeta_{\kappa} p^{t+r \kappa-1}-p^{t+r l-1}}\right) .
\end{aligned}
$$

Таким образом, если при всех $\vec{\zeta} \in \mathcal{I}(m, p)$

$$
\bigoplus_{\kappa=0, \ldots, m-1: \zeta_{\kappa}>0}\left(\xi_{0}^{-1} \xi_{1}\right)^{p^{t+r \kappa-1}} \neq 0
$$

и

$$
\bigoplus_{l=0, \ldots, m-1: \zeta_{l}>0} \gamma_{0}\left(\frac{\zeta_{l}}{\prod_{\kappa=0}^{m-1} \zeta_{\kappa} !}\right)\left(\theta_{0}^{-1} \theta_{1}\right)^{\sum_{\kappa=0}^{m-1} \zeta_{\kappa} p^{t+r \kappa-1}-p^{t+r l-1}} \neq 0,
$$

то минимальный многочлен последовательности (3.29) равен

$$
Z(x) \vee \underbrace{(x \ominus e)^{2} \vee \cdots \vee(x \ominus e)^{2}}_{\text {дизъюнкция } p-1 \text { сомножителей }}=Z(x)^{p} .
$$

Следовательно, при выписанных условиях минимальный многочлен последовательности, представленной второй суммой в равенстве $(3.24)$, равен $Z(x)^{p}$, и при этих же условиях $\epsilon_{b}^{(2)}=p$.

Рассмотрим минимальный многочлен последовательности, представленной первой суммой в (3.24). Как и ранее, нетрудно заметить, что эта последовательность представляется в виде суммы всех возможных последовательностей вида

$$
\left(\xi_{0} \theta_{0}^{i}\right)^{\sum_{l=0}^{m-1} p^{t+r l-2}\left(\lambda_{l}+p \zeta_{l}\right)} \cdot \gamma_{0}(i)^{\sum_{\kappa=m}^{2 m-1} \nu_{\kappa}}
$$

с некоторыми коэффициентами, не обязательно ненулевыми.

Следовательно, минимальный многочлен исследуемой последовательности равен многочлену

$$
\prod_{s=1}^{p-1} \check{H}_{s}(x)^{\beta_{s}}
$$

где параметры $\beta_{s}, s=1, \ldots, p-1$, определяются максимальным показателем степени при $\gamma_{0}(i)$, в которой это выражение входит в качестве сомножителя в суммируемые подпоследовательности, составляющие исследуемую последовательность, а многочлены $\check{H}_{s}(x)$ суть произведения сомножителей вида

$$
\left(x \ominus \theta_{0}^{\sum_{l=0}^{m-1} p^{t+r l-2}\left(\lambda_{l}+p \zeta_{l}\right)}\right),
$$


соответствующих слагаемым вида (3.33), входящим в первую сумму соотношения (3.24) с ненулевыми коэффициентами .

Исследуем вопрос о том, какое максимальное значение при фиксированных $\vec{\lambda} \in$ $\mathcal{I}(m, p), \vec{\zeta} \in \mathcal{I}(m, p-1)$ может принимать выражение $\sum_{\kappa=m}^{2 m-1} \nu_{\kappa}$.

Очевидно, что $\nu_{\kappa} \leqslant \zeta_{\kappa-m}, \kappa=m, \ldots, 2 m-1$, причем эта оценка достижима, и, следовательно, $\sum_{\kappa=m}^{2 m-1} \nu_{\kappa} \leqslant p-1$, причем эта оценка также достижима.

Предположим, что $\sum_{\kappa=m}^{2 m-1} \nu_{\kappa}=p-1$, и рассмотрим коэффициент при $\gamma_{0}(i)^{p-1}$ во внутренней части первой суммы равенства (3.24).

Заметим, что если $\sum_{\kappa=m}^{2 m-1} \nu_{\kappa}=p-1$ и $\nu_{2 m} \neq 0$, то $\nu_{0}=\ldots=\nu_{m-1}=0$ и $\nu_{2 m}=1$. Следовательно, в указанной сумме необходимо выделить подсумму, соответствующую значению $s=1$.

Поэтому искомый коэффициент равен

$$
\begin{aligned}
& \bigoplus_{\Psi(\tilde{b})} \gamma_{0}\left(\frac{1}{\prod_{\kappa=0}^{2 m-1} \nu_{\kappa} !} \cdot \frac{1}{\prod_{s=1}^{N} \eta_{s} !\left(\prod_{l=0}^{m-1} j_{l}^{(s)} !\right)^{\eta_{s}}}\right) \cdot\left(\xi_{0}^{-1} \xi_{1}\right)^{\sum_{\kappa=0}^{m-1} \nu_{\kappa} p^{t+r \kappa-1}} \cdot\left(\theta_{0}^{-1} \theta_{1}\right)^{\sum_{\kappa=m}^{2 m-1} \nu_{\kappa} p^{t+r \kappa-1}}= \\
& =\bigoplus_{\Psi(\tilde{b}, 1)} \gamma_{0}\left(\frac{1}{\prod_{\kappa=0}^{m-1} \nu_{\kappa} !}\right) \cdot\left(\theta_{0}^{-1} \theta_{1}\right)^{\sum_{\kappa=0}^{m-1} \nu_{\kappa} p^{t+r \kappa-1}} \cdot \gamma_{0}\left(\frac{1}{\prod_{s=1}^{\mathcal{N}}\left(\prod_{l=0}^{m-1} j_{l}^{(s) !}\right)^{\eta_{s}}}\right)= \\
& =\gamma_{0}\left(\frac{1}{\prod_{\kappa=0}^{m-1} \zeta_{\kappa} !}\right) \cdot\left(\theta_{0}^{-1} \theta_{1}\right)^{\sum_{\kappa=0}^{m-1} \zeta_{\kappa} p^{t+r \kappa-1}} \cdot \bigoplus_{\substack{s=1, N \\
j_{l}^{(s)}=\lambda_{l}, l=0, \ldots, m-1}} \gamma_{0}\left(\frac{1}{\prod_{l=0}^{m-1} j_{l}^{(s)} !}\right)= \\
& =\gamma_{0}\left(\frac{1}{\prod_{\kappa=0}^{m-1} \lambda_{\kappa} ! \zeta_{\kappa} !}\right) \cdot\left(\theta_{0}^{-1} \theta_{1}\right)^{\sum_{\kappa=0}^{m-1} \zeta_{\kappa} p^{t+r \kappa-1}},
\end{aligned}
$$

где

$$
\Psi(\tilde{b}, 1)=\left\{(\vec{\nu}, \vec{\eta}) \in \mathcal{I}(m, p-1) \times \mathcal{I}(\mathcal{N}, 1): \nu_{l}=\zeta_{l}, \sum_{s=1}^{\mathcal{N}} \eta_{s} j_{l}^{(s)}=\lambda_{l}, l \in\{0, \ldots, m-1\}\right\}
$$

то есть соответствующий коэффициент всегда ненулевой.

Таким образом, минимальный многочлен последовательности, представленной первой суммой в правой части равенства (3.24), как следует из леммы 2.4 и результатов работы [17], делится на многочлен

$$
H(x) \vee \underbrace{(x \ominus e)^{2} \vee \cdots \vee(x \ominus e)^{2}}_{\text {дизъюнкция } p-1 \text { сомножителей }}=H(x)^{p} .
$$


Итак, при условиях (3.30), (3.31) минимальный многочлен последовательности $\tilde{b}$ равен

$$
Z(x)^{p} \cdot H(x)^{p} \cdot \bar{F}(x)^{p} \cdot \prod_{s=2}^{p-1} \check{H}_{s}(x)^{\beta_{s}}, \text { где } 0 \leqslant \beta_{s} \leqslant p-1, s=2, \ldots, p-1 .
$$

Если эти условия не выполнены, то минимальный многочлен последовательности, представленной второй суммой в равенстве (3.24), имеет значительно более сложный вид, и в этом случае можно лишь утверждать, что

$$
H(x)^{p} \cdot \bar{F}(x)^{p}\left|m_{\tilde{b}}(x)\right| Z(x)^{p} \cdot H(x)^{p} \cdot \bar{F}(x)^{p} \cdot \prod_{s=2}^{p-1} H_{s}(x)^{\beta_{s}},
$$

где $0 \leqslant \beta_{s} \leqslant p-1, s=2, \ldots, p-1$.

Пусть теперь $\xi_{1}=0$ и $\theta_{1} \neq 0$.

Тогда, повторяя выкладки (3.17)-(3.24), получим:

$$
\begin{gathered}
\tilde{b}(i)=\bigoplus_{s=1}^{p-1} \bigoplus_{\substack{\vec{\lambda} \in \mathcal{I}(m, p s), \vec{\zeta} \in \mathcal{I}(m, p-s)}}\left(\xi_{0} \theta_{0}^{i}\right)^{\sum_{l=0}^{m-1} p^{t+r l-2}\left(\lambda_{l}+p \zeta_{l}\right)} \times \\
\times\left(\bigoplus_{\Psi(\tilde{b}, 2)} \gamma_{0}\left(\frac{1}{\prod_{\kappa=0}^{m-1} \nu_{\kappa} !} \frac{1}{\prod_{s=1}^{\mathcal{N}} \eta_{s} !\left(\prod_{l=0}^{m-1} j_{l}^{(s)} !\right)^{\eta_{s}}}\right)\left(\theta_{0}^{-1} \theta_{1}\right)^{\sum_{\kappa=0}^{m-1} \nu_{\kappa} p^{t+r \kappa-1} \gamma_{0}(i)^{\sum_{\kappa=0}^{m-1} \nu_{\kappa}}}\right) \oplus \\
\oplus \underset{\vec{\zeta} \in \mathcal{I}(m, p)}{\bigoplus}\left(\xi_{0} \theta_{0}^{i}\right)^{\sum_{l=0}^{m-1} p^{t+r l-1} \zeta_{l}} \cdot \gamma_{0}\left(\frac{1}{\prod_{l=0}^{m-1} \zeta_{l} !}\right) \cdot \gamma_{0}(i) \cdot\left(\theta_{0}^{-1} \theta_{1}\right)^{\sum_{l=0}^{m-1} \zeta_{l} p^{t+r l-1}}, \quad(3.36)
\end{gathered}
$$

где

$$
\begin{aligned}
& \Psi(\tilde{b}, 2)=\left\{(\vec{\nu}, \vec{\eta}) \in \mathcal{I}(m+1, p) \times \mathcal{I}\left(\mathcal{N}, \nu_{m}\right):\right. \\
& \left.\nu_{m} \neq 0, p \nu_{l}+\sum_{s=1}^{\mathcal{N}} \eta_{s} j_{l}^{(s)}=\lambda_{l}+p \zeta_{l}, l \in\{0, \ldots, m-1\}\right\} .
\end{aligned}
$$

Минимальный многочлен последовательности, представленной второй суммой в равенстве (3.36), равен $Z(x)^{2}$.

Как и выше, максимальное значение, которое может принимать показатель степени при $\gamma_{0}(i)$ в последовательности, представленной первой суммой в равенстве (3.36), равен $p-1$, и коэффициент при $\gamma_{0}(i)^{p-1}$ при фиксированных векторах $\vec{\lambda} \in \mathcal{I}(m, p), \vec{\zeta} \in \mathcal{I}(m, p-1)$ равен

$$
\gamma_{0}\left(\frac{1}{\prod_{l=0}^{m-1} \lambda_{l} ! \zeta_{l} !}\right) \cdot\left(\theta_{0}^{-1} \theta_{1}\right)^{\sum_{\kappa=0}^{m-1} \zeta_{\kappa} p^{t+r \kappa-1}} .
$$

Следовательно, если $\xi_{1}=0$, то минимальный многочлен последовательности $\tilde{b}$ удовлетворяет условиям

$$
H(x)^{p} \cdot Z(x)^{2}\left|m_{\tilde{b}}(x)\right| H(x)^{p} \cdot Z(x)^{2} \cdot \prod_{s=2}^{p-1} H_{s}(x)^{\beta_{s}}
$$


где $0 \leqslant \beta_{s} \leqslant p-1, s=2, \ldots, p-1$.

Итак, с учетом соотношения (3.7), лемм $3.1,3.2,3.3$, а также того, что многочлен $G(x)$ делит многочлен $Z(x)$ и последовательности $\tilde{b}$ и $\gamma_{2}(c)$ различны, окончательно получаем утверждение теоремы 3.1 .

Утверждение 3.1. В условиях теоремы 3.1 каждый элемент вида

$$
\theta_{0}^{\sum_{l=0}^{m-1} p^{r m+r l-2}\left(\lambda_{l}+p \zeta_{l}\right)}, \vec{\lambda} \in \mathcal{I}(m, p), \vec{\zeta} \in \mathcal{I}(m, p-1),
$$

является корнем многочлена $m_{2}(x)$ кратности $р$.

Утверждение 3.2. I. В условиях теоремы 3.1 выполнены неравенства

$$
\begin{aligned}
m(p+1) & +p\left\{\begin{array}{c}
m \\
p
\end{array}\right\}\left\{\begin{array}{c}
m \\
p-1
\end{array}\right\} \leqslant \operatorname{rank} u_{2} \leqslant \\
& \leqslant m(p+1)+p\left\{\begin{array}{c}
m \\
p
\end{array}\right\}\left\{\begin{array}{c}
m \\
p-1
\end{array}\right\}+(p-1) \sum_{s=2}^{p-1}\left\{\begin{array}{c}
m \\
p s
\end{array}\right\}\left\{\begin{array}{c}
m \\
p-s
\end{array}\right\}+p\left\{\begin{array}{c}
m \\
p
\end{array}\right\}+\left\{\begin{array}{c}
m \\
p^{2}
\end{array}\right\} .
\end{aligned}
$$

II. Если выполнены условия теоремы 3.1 и при всех $\vec{\zeta} \in \mathcal{I}(m, p)$

$$
\bigoplus_{\kappa=0, \ldots, m-1: \zeta_{\kappa}>0}\left(\xi_{0}^{-1} \xi_{1}\right)^{p^{t+r \kappa-1}} \neq 0
$$

$u$

$$
\bigoplus_{l=0, \ldots, m-1: \zeta_{l}>0} \gamma_{0}\left(\frac{\zeta_{l}}{\prod_{\kappa=0}^{m-1} \zeta_{\kappa} !}\right)\left(\theta_{0}^{-1} \theta_{1}\right)^{\sum_{\kappa=0}^{m-1} \zeta_{\kappa} p^{t+r \kappa-1}-p^{t+r l-1}} \neq 0
$$

mo

$$
\begin{aligned}
m(p+1)+p\left\{\begin{array}{c}
m \\
p
\end{array}\right\}\left\{\begin{array}{c}
m \\
p-1
\end{array}\right\}+p\left\{\begin{array}{c}
m \\
p
\end{array}\right\} \leqslant \operatorname{rank} u_{2} \leqslant \\
\leqslant m(p+1)+p\left\{\begin{array}{c}
m \\
p
\end{array}\right\}\left\{\begin{array}{c}
m \\
p-1
\end{array}\right\}+(p-1) \sum_{s=2}^{p-1}\left\{\begin{array}{c}
m \\
p s
\end{array}\right\}\left\{\begin{array}{c}
m \\
p-s
\end{array}\right\}+\left\{\begin{array}{c}
m \\
p^{2}
\end{array}\right\} .
\end{aligned}
$$

III. В условиях теоремы 3.1 при $\xi_{1}=0$ имеют место неравенства

$$
\begin{aligned}
m(p+1)+p\left\{\begin{array}{c}
m \\
p
\end{array}\right\} & \left\{\begin{array}{c}
m \\
p-1
\end{array}\right\}+2 \cdot\left\{\begin{array}{c}
m \\
p
\end{array}\right\} \leqslant \operatorname{rank} u_{2} \leqslant \\
& \leqslant m(p+1)+p\left\{\begin{array}{c}
m \\
p
\end{array}\right\}\left\{\begin{array}{c}
m \\
p-1
\end{array}\right\}+(p-1) \sum_{s=2}^{p-1}\left\{\begin{array}{c}
m \\
p s
\end{array}\right\}\left\{\begin{array}{c}
m \\
p-s
\end{array}\right\}+\left\{\begin{array}{c}
m \\
p^{2}
\end{array}\right\} .
\end{aligned}
$$

Замечание 3.3. Согласно [11] при условиях теоремы 3.1 имеет место неравенство

$$
m(p+1) \leqslant \operatorname{rank} u_{2} .
$$

Таким образом, оценки утверждения 3.2 уточняют ранее полученные результаты.

Автор искренне признателен В.Л. Куракину за оказанную помощь и внимание к работе. 


\section{Список литературы}

1. Берлекэмп Э.Р.Алгебраическая теория кодирования, М.: "Мир", 1971, 568 с.; пер. с англ.: Berlekamp E. R., Algebraic coding theory, McGraw-Hill, 1968, 466 c.

2. Куракин В.Л., "Представления над кольцом $\mathbb{Z} / p^{n}$ линейной рекуррентной последовательности максимального периода над полем $G F(p)$ ", Дискретная математика, 4:4 (1992), 96-116; англ. пер.: Kurakin V.L., "Representations over $\mathbb{Z}_{p^{n}}$ of linear recurring sequence of maximal period over GF(p)", Discrete Math. Appl., 3:3 (1993), 275-296.

3. Куракин В.Л., "Первая координатная последовательность линейной рекурренты максимального периода над кольцом Галуа", Дискретная математика, 6:2 (1994), 88-100; англ. пер.: Kurakin V.L., "The first coordinate sequence of a linear recurrence of maximal period over a Galois ring", Discrete Math. Appl., 4:2 (1994), 129-141.

4. Лидл Р., Нидеррайтер Г., Конечные поля, mm. 1,2, М.: "Мир", 1988, 819 с.; пер. с англ.: Lidl R., Niederreiter H., "Finite fields", Encyclopedia of Mathematics and its Applications, 20, Addison-Wesley, 1987, 755 pp.

5. Нечаев А.А., "Код Кердока в циклической форме", Дискретная математика, 1:4 (1989), 123-139; англ. пер.: Nechaev A.A., "Kerdock code in a cyclic form", Discrete Math. Appl., 1:4 (1991), 365-384.

6. Нечаев А.А., "Линейные рекуррентные последовательности над коммутативными кольцами", Дискретная математика, 3:4 (1991), 107-121; англ. пер.: "Linear recurrence sequences over commutative rings", Discrete Math. Appl., 2:6 (1992), 659-683.

7. Сачков В.Н., Введение в комбинаторные методы дискретной математики, М.: Наука, 1982, 384 с.

8. Цыпышев В. Н., "Матричный линейный конгруэнтный генератор над кольцом Галуа нечетной характеристики", Тезисы докл. V междунар. конф. "Алгебра и теория чисел: Современные проблемы и приложения" (Тула, 2003), 2003, 233-237.

9. Цыпышев В.Н., "Критерий полноты периода многочлена Галуа над собственным кольцом Галуа нечетной характеристики", Современная математика и ее приложения, 14 (2004), 108-120.

10. Helleseth T., Martinsen M., "Binary sequences of period $2^{m}-1$ with large linear complexity", Inf. and Comput., 151 (1999), 73-91.

11. Kuzmin A.S., Nechaev A.A., "Linear recurrent sequences over Galois rings", II Int.Conf.Dedic.Mem. A.L.Shirshov (Barnaul), Contemporary Math., 184 (1995), 237-254.

12. McDonald C., Finite rings with identity, New York: Marcel Dekker, 1974, 495 pp.

13. Raghavendran R., "A class of finite rings", Compositio Math., 22:1 (1970), 49-57.

14. Цыпышев В. Н., "Критерий полноты периода многочлена Галуа над невырожденным кольцом Галуа нечетной характеристики", Современная математика и ее приложения, 2004, № 14, 108-120; англ. пер.: Tsypyschev, V.N., "Full periodicity of Galois polynomials over nontrivial Galois rings of odd characteristic", J. Math. Sci., 131:6 (2005), 6120-6132.

15. Tsypyschev V.N., "Rank estimations of the second coordinate sequence of MP-LRS over nontrivial Galois ring of odd characteristic", II Int. Sci. Conference on Problems of Security and Counter-Terrorism Activity (Moscow, MSU, October 25-26, 2006), 2007, 287-289 (in Russian).

16. Tsypyschev V.N. Second coordinate sequence of the MP-LRS over nontrivial Galois ring of the odd characteristic, IV Международный симпозиум "Современные тенденции в криптографии" CtCrypt'2015 (Казань, 3-5 июня 2015 г.), Материаль конференции.

17. Zierler N., Mills W., "Products of linear recurring sequences", J. Algebra, 27 (1973), $147-157$. 